\title{
MYB controls erythroid versus megakaryocyte lineage fate decision through the miR-486-3p-mediated downregulation of MAF
}

\author{
E Bianchi ${ }^{1}$, J Bulgarelli ${ }^{1}$, S Ruberti ${ }^{1}$, S Rontauroli ${ }^{1}$, G Sacchi ${ }^{1}$, R Norfo ${ }^{1}$, V Pennucci ${ }^{1}$, R Zini ${ }^{1}$, S Salati ${ }^{1}$, Z Prudente ${ }^{1}$, S Ferrari ${ }^{2}$ \\ and R Manfredini*,
}

The transcription factor MYB has a key role in hematopoietic progenitor cells (HPCs) lineage choice, by enhancing erythropoiesis at the expense of megakaryopoiesis. We previously demonstrated that MYB controls erythroid versus megakaryocyte lineage decision by transactivating KLF1 and LMO2 expression. To further unravel the molecular mechanisms through which MYB affects lineage fate decision, we performed the integrative analysis of miRNA and mRNA changes in MYB-silenced human primary CD34+ HPCs. Among the miRNAs with the highest number of predicted targets, we focused our studies on hsa-miR-486-3p by demonstrating that MYB controls miR-486-3p expression through the transactivation of its host gene, ankyrin-1 (ANK1) and that miR-486-3p affects HPCs commitment. Indeed, overexpression and knockdown experiments demonstrated that miR-486-3p supports the erythropoiesis while restraining the megakaryopoiesis. Of note, miR-486-3p also favors granulocyte differentiation while repressing the macrophage differentiation. To shed some light on the molecular mechanisms through which miR-486-3p affects HPCs lineage commitment, we profiled the gene expression changes upon miR-486-3p overexpression in CD34+ cells. Among the genes downregulated in miR-486-3p-overexpressing HPCs and computationally predicted to be miR-486-3p targets, we identified MAF as a miR-486-3p target by $3^{\prime}$ UTR luciferase reporter assay. Noteworthy, MAF overexpression was able to partially reverse the effects of miR-486-3p overexpression on erythroid versus megakaryocyte lineage choice. Moreover, the MYB/MAF co-silencing constrained the skewing of erythroid versus megakaryocyte lineage commitment in MYB-silenced CD34+ cells, by restraining the expansion of megakaryocyte lineage while partially rescuing the impairment of erythropoiesis. Therefore, our data collectively demonstrate that MYB favors erythropoiesis and restrains megakaryopoiesis through the transactivation of miR-486$3 p$ expression and the subsequent downregulation of MAF. As a whole, our study uncovers the MYB/miR-486-3p/MAF axis as a new mechanism underlying the MYB-driven control of erythroid versus megakaryocyte lineage fate decision.

Cell Death and Differentiation (2015) 22, 1906-1921; doi:10.1038/cdd.2015.30; published online 10 April 2015

The transcription factor MYB ( $v$-myb avian myeloblastosis viral oncogene homolog, $c-m y b)$ has a pivotal role in the hematopoietic system development and adult hematopoietic progenitor cells (HPCs) lineage commitment, as demonstrated by in vivo knockout/knockdown models ${ }^{1,2}$ and in vitro silencing approaches. ${ }^{3}$

Despite the broad bibliography dealing with the function of MYB in hematopoiesis, the molecular mechanisms underlying the role of this transcription factor in hematopoietic differentiation are still partially unknown. More in detail, several in vivo models demonstrate that the knockout/knockdown of MYB impairs erythropoiesis ${ }^{1,2}$ and the reduction of its transcriptional activity causes an abnormal expansion of magakaryocytopoiesis. ${ }^{4-6}$ Nevertheless, the transcriptional targets of MYB that could explain these effects still remain uncovered. For this purpose, we previously demonstrated that
MYB affects erythroid versus megakaryocyte commitment by transactivating the expression of the transcription factors $\mathrm{KLF}^{7,8}$ and $\mathrm{LMO} 2$. $^{3,9}$ At the same time, gene expression profiling (GEP) data from MYB-silenced hematopoietic progenitor cells (HPCs) highlighted the upregulation of several transcription factors having a pivotal role in hematopoiesis and potentially involved in MYB-directed HPCs lineage choice, such as MAF, ${ }^{10,11}$ MAFB $^{12}$ and MEIS1. ${ }^{13}$ However, ChIP data showed that these genes are not direct transcriptional targets of MYB, ${ }^{3}$ that is, MYB is unable to directly bind their promoter regions by repressing their expression. Therefore, the mechanisms through which they are modulated upon MYB silencing remains to be clarified.

For this purpose, we decided to unravel the microRNA (miRNA) and the mRNA expression changes induced by MYB silencing in cord blood (CB)-derived CD34+ HPCs.

\footnotetext{
${ }^{1}$ Department of Life Sciences, Center for Regenerative Medicine 'Stefano Ferrari', University of Modena and Reggio Emilia, Modena, Italy and ${ }^{2}$ Department of Life Sciences, Center for Genome Research, University of Modena and Reggio Emilia, Modena, Italy

*Corresponding author: R Manfredini, Department of Life Sciences, Center for Regenerative Medicine 'Stefano Ferrari', University of Modena and Reggio Emilia, via Gottardi 100, 41125 Modena, Italy. Tel: +39 059 2058065; Fax: +39 059 2058116; E-mail: manfredini.rossella@unimore.it

Abbreviations: MYB, v-myb avian myeloblastosis viral oncogene homolog, c-myb; HPCs, Hematopoietic Progenitor Cells; CB, cord blood; GEP, gene expression profiling; miEP, miRNA expression profiling; DEGs, differentially expressed genes; DEMs, differentially expressed miRNA; GEO, Gene Expression Omnibus; IPA, Ingenuity Pathway Analysis; ANK1, ankyrin-1; TSS, transcription start site; ChIP, Chromatin Immunoprecipitation; CFU-MK, Megakaryocyte colony forming unit; MGG, May Grunwald-Giemsa; 3'UTR, 3'-untranslated region; MAF, v-maf musculoaponeurotic fibrosarcoma oncogene homolog, avian, c-maf

Received 14.8.14; revised 23.1.15; accepted 20.2.15; Edited by R De Maria; published online 10.4.15
} 
Indeed, increasing evidences stress the key role of miRNAs in controlling many cellular processes including hematopoietic lineage commitment ${ }^{14,15}$ through the post-transcriptional repression of their target genes, ${ }^{16}$ mainly by mRNA degradation or inhibition of protein translation.

In order to unveil whether the MYB-regulated miRNAs could further exlplain the role of MYB in promoting erythropoiesis and repressing megakaryopoiesis, GEP and miRNA expression profiling (miEP) data from MYB-silenced HPCs were integrated in silico by using QIAGEN's Ingenuity Pathway Analysis Software (IPA, QIAGEN, Redwood City, CA, USA; www.qiagen.com/ingenuity). IPA analysis disclosed 242 miRNA-mRNA pairs differentially expressed in MYBsilenced compared with control CD34+ cells and with anticorrelated expression trend. Among the miRNAs with the highest number of putative targets, that is, mRNAs having an anti-correlated expression pattern, we focused our attention on hsa-miR-486-3p (hereafter reported as miR-486-3p) as downregulated upon MYB silencing together with the host gene ankyrin-1 (ANK1). Our data demonstrate that MYB affects HPCs fate decision through the transcriptional control of miR-486-3p expression.

\section{Results}

mRNA and miRNA expression profiling in CD34+ HPCs upon MYB silencing. To shed some light on the MYB-driven miRNA-mediated regulation of gene expression during HPCs lineage commitment, we profiled the mRNA and miRNA expression changes induced by MYB silencing in human CD34+ cells. RNAi-mediated experiments were performed by nucleofection of MYB-targeting siRNAs (Supplementary Table S1), as previously detailed. ${ }^{3,17}$ For each experiment, one sample transfected with a non-targeting siRNA as a negative control (NegCTRsiRNA) was performed besides the MYB-targeting siRNA-transfected sample (MYBsiRNA).
As previously shown, ${ }^{3}$ in these experimental conditions the best downregulation of MYB protein levels is achieved at $24 \mathrm{~h}$ after the last of three 24 hours spaced nucleofection cycles (hereafter reported as post nucleofection) in CD34+ cells; therefore, we selected this time point for studying the mRNA and miRNA expression changes induced by MYB silencing during HPCs commitment. In a set of five independent experiments, MYBsiRNA and NegCTR CD34+ cells were profiled for mRNA and miRNA expression by Affymetrix U219 Array (Affymetrix; Santa Clara, CA, USA) and Exiqon Human miRNome PCR Panel (Exiqon, Copenhagen, Denmark), respectively. GEP and miEP analysis disclosed 616 DEGs and 37 differentially expressed miRNAs (DEMs) between MYBsiRNA and NegCTRsiRNA samples (Supplementary Tables S2 and S3; DEGs upon MYB silencing were also profiled at 24 hours after one and two nucleofection cycles: GEP data were deposited in the GEO repository; see the Materials and Methods section).

Next, in order to build the regulatory networks between the miRNAs and their predicted targets modulated by MYB silencing, in silico integrative analysis by means of IPA was performed. IPA analysis highlighted 20 DEMs with at least one anti-correlated target among DEGs, and 150 DEGs with at least one anti-correlated targeting DEM, overall describing 242 miRNA-mRNA pairs with anti-correlated expression trend (Supplementary Table S4).

Among the 20 DEMs from IPA analysis, we focused our attention on hsa-miR-486-3p as one of the miRNAs downregulated upon MYB silencing and displaying the highest number of putative targets (Table 1, Supplementary Figure S1). Notably, miR-486-3p is an intragenic (intronic) miRNA (Figure 1a) whose host gene, ankyrin-1 (ANK1) was similarly downregulated upon MYB silencing (Supplementary Table S2).

MYB-dependent transcriptional control of ANK1 and miR-486-3p. To get further insights into the relationship

Table 1 DEMs from integrated miRNA-mRNA analysis on MYB-silenced CD34+ HPCs

\begin{tabular}{|c|c|c|c|}
\hline miRNA ID & miRNA family & $\begin{array}{l}\text { Fold Change in MYB-silenced } \\
\text { versus control CD34+ cells }\end{array}$ & $\begin{array}{c}\text { Number of anti-correlated } \\
\text { putative targets }\end{array}$ \\
\hline hsa-miR-144-3p & miR-144-3p (miRNAs w/seed ACAGUAU) & -3.245 & 31 \\
\hline hsa-miR-486-3p & miR-486-3p (and other miRNAs w/seed GGGGCAG) & -3.201 & 26 \\
\hline hsa-miR-152 & miR-148b-3p (and other miRNAs w/seed CAGUGCA) & -1.972 & 24 \\
\hline hsa-miR-92b-3p & miR-92a-3p (and other miRNAs w/seed AUUGCAC) & -2.542 & 19 \\
\hline hsa-miR-491-5p & miR-491-5p (and other miRNAs w/seed GUGGGGA) & -1.647 & 16 \\
\hline hsa-miR-671-5p & miR-671-5p (miRNAs w/seed GGAAGCC) & -2.844 & 16 \\
\hline hsa-miR-197-3p & miR-197-3p (and other miRNAs w/seed ÚCACCAC) & -1.625 & 14 \\
\hline hsa-miR-339-5p & miR-339-5p (and other miRNAs w/seed CCCUGUC) & -1.524 & 14 \\
\hline hsa-miR-545-3p & miR-545-3p (miRNAs w/seed CAGCAAA) & -2.336 & 11 \\
\hline hsa-miR-520h & miR-520g-3p (and other miRNAs w/seed CAAAGUG) & -2.564 & 10 \\
\hline hsa-miR-877-5p & miR-877-5p (and other miRNAs w/seed UAGAGGA) & -2.003 & 10 \\
\hline hsa-miR-296-3p & miR-296-3p (miRNAs w/seed AGGGUUG) & -4.443 & 9 \\
\hline hsa-miR-362-5p & miR-362-5p (and other miRNAs w/seed AUCCUUG) & -1.701 & 9 \\
\hline hsa-miR-151a-3p & miR-151-3p (and other miRNAs w/seed UAGACUG) & -1.989 & 8 \\
\hline hsa-miR-335-5p & miR-335-5p (and other miRNAs w/seed CAAGAGC) & 1.516 & 8 \\
\hline hsa miR-486-5p & miR-486-5p (and other miRNAs w/seed CCUGUAC) & -3.002 & 6 \\
\hline hsa-miR-151a-5p & miR-151-5p (and other miRNAs w/seed CGAGGAG) & -1.571 & 5 \\
\hline hsa-miR-376b-3p & miR-376a-3p (and other miRNAs w/seed UCAUAGA) & 1.625 & 5 \\
\hline hsa-miR-191-5p & miR-191-5p (and other miRNAs w/seed AACGGAA) & -1.696 & 2 \\
\hline hsa-miR-337-5p & miR-337-5p (miRNAs w/seed AACGGCU) & -1.643 & 2 \\
\hline
\end{tabular}

Abbreviations: DEMs, differentially expressed miRNAs; HPCs, hematopoietic progenitor cells; miRNA, microRNA 
a

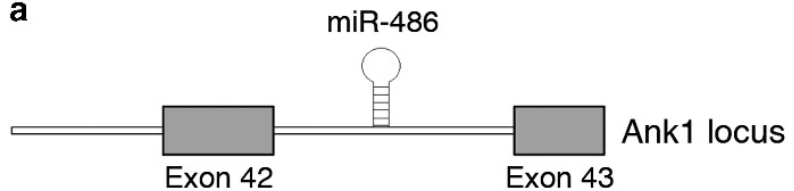

b

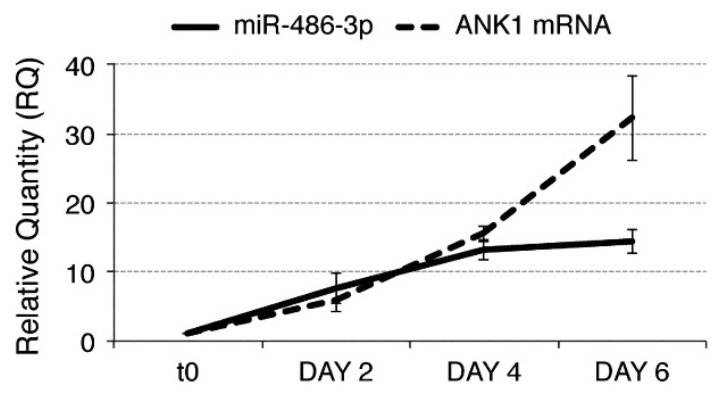

C

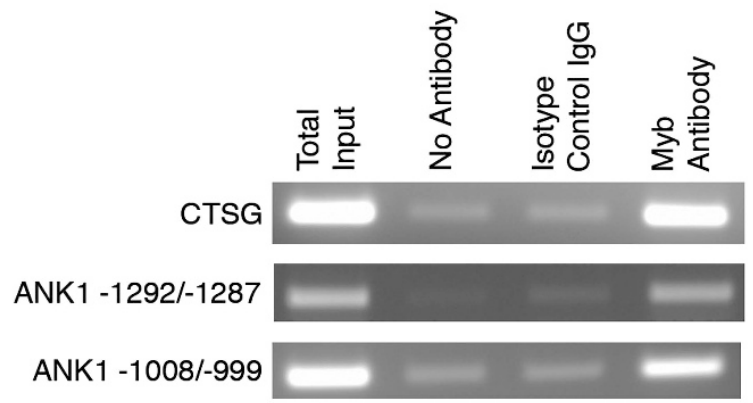

d

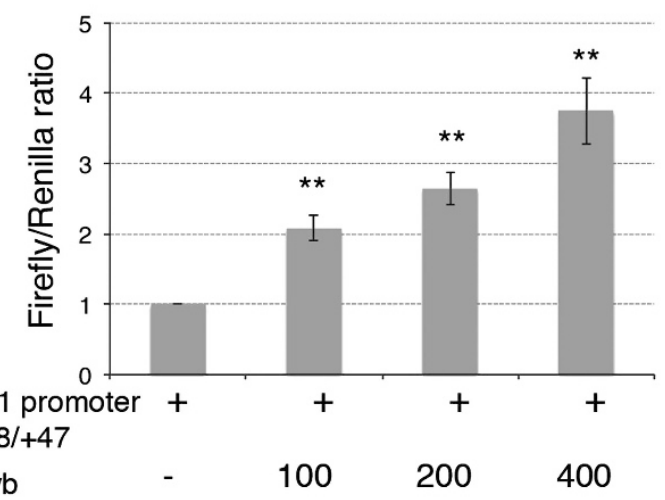

Figure 1 MYB-driven regulation of the ANK1 gene and the embedded miR-486$3 p$ expression. (a) miR-486 is under the transcriptional regulation of the ankyrin 1 (ANK1) locus; data from UCSC Human Genome Browser-hg19 assembly. (b) miR-486-3p and ANK1 expression kinetics during erythroid differentiation of CD34 + cells. The expression levels of miR-486-3p and ANK1 mRNA were monitored by qRTPCR at different culture time points after the EPO-driven induction of the erythroid differentiation in CD34+ cells. miR-486-3p mature miRNA and ANK1 mRNA levels were normalized versus housekeeping U6 snRNA and HPRT1 mRNA, respectively, and are reported as $R Q$ respect to $C D 34+$ cells at time 0 of treatment with $E P O$, which was set as calibrator. $P \leq 0.05$ at day 4 and day 6 compared with to for both miR-486-3p and ANK1. (c) ANK1 promoter-specific PCR performed in a representative ChIP experiment. PCR data are reported for both the putative MYB binding sites with the highest computational prediction scores (i.e. -1292 - 1287 and - 1008/ - 999, see Supplementary Table S5 for further details). ChIP and PCRs were performed in triplicate. (d) MYB transactivation of ANK1 promoter-driven luciferase expression. The amounts (in ng) of co-transfected plasmids are reported. Renilla-normalized Firefly luciferase levels (mean \pm S.E.M.; $n=5)$ are normalized by setting the pXP1-ANK1(-1498/+47)/pCMV6XL5 empty vector-transfected sample as $=1$. Error bars represent S.E.M. ${ }^{* *} P \leq 0.01$ compared with pXP1ANK1(-1498/+47)/pCMV6XL5 empty vector-transfected sample. $n=$ number of experiments between the silencing of MYB and the downregulation of miR-486-3p/ANK1, we detected the kinetics of miR-486-3p and ANK1 mRNA expression during HPCs erythroid differentiation. We found that both miR-486-3p and the ANK1 were strongly upregulated during erythroid differentiation (Figure 1b), further supporting the notion that miR-486-3p expression is regulated by the promoter region of the host ANK1 gene. ${ }^{18-20}$

Next, we screened the ANK1 promoter region for putative MYB binding sites by matching the genomic sequence upstream the ANK1 gene transcription starting site (TSS) to the TRANSFAC consensus-binding sequences ${ }^{21}$ (http://www. cbrc.jp/research/db/TFSEARCH.html). Computational screening highlighted the presence of two putative binding sites for MYB with high scores (based on the TRANSFAC positional weight matrix for MYB) localized within 2000 bps upstream the ANK1 gene TSS (Supplementary Table S5). Therefore, we checked the ANK1 promoter region for the direct binding of MYB by Chromatin Immunoprecipitation (ChIP). ${ }^{3}$

ChIP experiments in CD34+ cells demonstrated the direct binding of MYB to the ANK1 promoter in vivo. In fact, MYB antibody-mediated immunoprecipitation resulted in amplification of the ANK1 promoter region, while immunoprecipitation without antibody or with an isotype-matched control antibody determined a negligible amplification (Figure 1c).

In addition, luciferase reporter assays demonstrated that MYB is able to transactivate ANK1 promoter, as increasing doses of MYB-coding vector caused a dose-dependent increase in ANK1 promoter-driven luciferase expression (Figure 1d).

Overall, these data indicated that MYB is able to bind to ANK1 promoter and to transactivate the expression of both the ANK1 gene and the embedded miR-486-3p.

miR-486-3p affects erythroid versus megakaryocyte lineage fate decision in HPCs. In order to understand whether miR-486-3p could be relevant for MYB-driven HPCs fate decision, we studied the effects of its overexpression by means of miR-486-3p mimic transfection (miR-486-3p mimic), compared with a negative control mimic-transfected sample (NegCTR mimic), in CB CD34+ cells. Similarly, we assayed the effects of miR-486-3p knockdown by the nucleofection of miR-486-3p and non-targeting control inhibitors (miR-486-3plnh/NegCTRInh) in CD34+ cells. Furthermore, in order to clarify whether the MYB-dependent miR-486-3p expression could be relevant in MYB-mediated control of erythroid versus megakaryocyte lineage fate decision, we studied the effects of the rescue of miR-486-3p expression in MYB-silenced HPCs through the co-transfection of MYB-targeting siRNAs and miR-486-3p mimics in CD34+ cells.

The effects of miR-486-3p overexpression and knockdown on CD34+ cells commitment were evaluated by flowcytometric analysis of differentiation markers and morphological analysis of May-Grunwald-Giemsa- (MGG) stained cytospins in unilineage megakaryocyte, erythroid, granulocyte and mono-macrophage cultures (Figures 2-4).

Our data demonstrated that miR-486-3p overexpression restrains megakaryocyte differentiation. Indeed, flow cytometry data highlighted a reduction of the megakaryocytecommitted population from the earlier CD34+CD41+ to the 
more mature CD34-CD41+, CD41+CD42b - and CD41+ CD42b+ fractions in miR486-3p mimic compared with NegCTR mimic cells at 4 days of culture (Figure 2a, Supplementary Figure S2Ai). The mature megakaryocytic CD41+CD42b+ population was similarly reduced upon miR-486-3p overexpression at later stages (Figure $2 b$, Supplementary Figures S2Aii-iii and S2B). Consistently, morphological analysis highlighted the impairment of megakaryocyte differentiation in miR-486-3p mimic (Figure 2ci) compared with NegCTR mimic sample (Figure 2cii). The perturbation of megakaryopoiesis was further demonstrated by the striking reduction in megakaryocyte hyperploid and proplatelet-producing cells detected in miR-486-3p mimic compared with NegCTR mimic cells in culture (Figure 2d).

We also studied the effects of miR-486-3p overexpression on megakaryocyte commitment by plating miR-486-3p and NegCTR mimic CD34+ cells in a collagen-based serum-free semisolid medium supporting the megakaryopoiesis in vitro. This assay demonstrated that miR-486-3p overexpression strongly represses the megakaryocyte commitment. Notably, the megakaryocyte colony forming units (CFU-MKs) scoring showed remarkable differences in medium and small CFUMKs between miR-486-3p mimic and NegCTR samples (Figure 2e, Supplementary Figure S2C), further demonstrating that miR-486-3p overexpression affects both early and late steps of megakaryocyte differentiation.

The effects of miR-486-3p knockdown were similarly assessed, by highlighting an increase in megakaryocyte differentiation at both early (CD34+CD41+ fraction, Figure 2f) and late differentiation steps (CD41+CD42b- and CD $41+C D 42 b+$ fractions at 8 and 12 days, Figures $2 g$ and $h$ ). The same trend was confirmed by the increase in CFU-MKs in miR-486-3plnh compared with NegCTRInh sample (Figure 2i).

Finally, MYB-targeting siRNAs/miR-486-3p mimics co-transfection experiments showed a reduction of both early CD34+CD41+ (Figure 2j) and more differentiated CD41+ CD42b+ population (Figures 2j and k) in MYBsiRNA/miR-486$3 p$ mimic compared with MYBsiRNA/NegCTR mimic sample. Similarly, a remarkable CFU-MKs reduction was detected in MYBsiRNA/miR-486-3p mimic compared with MYBsiRNA/ NegCTR mimic sample (Figure 2l).

Next, we studied the effects of miR-486-3p overexpression in CD34+ cells erythroid differentiation, by demonstrating that miR-486-3p enhances the erythropoiesis. Indeed, miR-486-3p overexpression caused an early increase in the erythroid CD36+GPA+ fraction (Figure 3a), followed by an expansion of the more mature CD36-GPA+ population at the later stages of erythroid differentiation (Figure 3a, Supplementary Figure S2D). In line with these data, morphological analysis higlighted an enrichment of orthochromatic erythroblasts with pyknotic nuclei and reticulocytes representing the last stages of erythropoiesis in miR-486-3p mimic samples (Figure 3bii), while NegCTR mimic samples were mainly represented by more immature cells, such as proerythroblasts and basophilic erythroblasts (Figure 3bi). Conversely, miR-486-3p knockdown in HPCs was able to negatively interfere with the erythroid differentiation, as demonstrated by the reduction in the CD36+GPA - and CD36+GPA+ fractions at 4 days post nucleofection (Figure $3 c$ ), and the subsequent decrease in
CD36+GPA+ and CD36-GPA+ populations at 8 and 12 days, respectively (Figures $3 d$ and $e$ ).

Noteworthy, the arrest in the early CD71+CD36-/CD71+ CD36+ transition and in the late CD36+GPA+/CD36-GPA+ maturation steps of erythropoiesis (Figures $3 f$ and g; MYBsiRNA/ NegCTR mimic sample) induced by MYB silencing was partially reversed by the miR-486-3p mimic co-transfection (Figures $3 f$ and g; MYBsiRNA/miR-486-3p mimic sample).

Collectively these results demonstrate that the MYB-driven miR-486-3p expression during HPCs commitment favors erythroid differentiation while restraining the megakaryocyte differentiation.

miR-486-3p favors granulocyte differentiation while repressing macrophage differentiation in HPCs. We further investigated the effects of miR-486-3p overexpression and knockdown in HPCs granulocyte and mono-macrophage differentiation by morphological and immunophenotypic analyses (Figure 4).

These experiments allowed us to demonstrate that miR-486-3p expression promotes granulocyte differentiation, while suppressing macrophage differentiation. Indeed, an increase in granulocyte lineage-specific MPO+ and CD15+ populations (Figure 4a, Supplementary Figure S2E) was detected in miR-486-3p mimic compared with NegCTR mimic sample during the granulocyte differentiation. In addition, morphological analysis clearly displayed an enrichment of cells at the later stages of granulocyte differentiation in miR-486-3p-overexpressing samples (Figure 4cii, iv) compared with the controls, still showing a higher fraction of more immature cells (Figure 4ci, iii).

Moreover, miR-486-3p overexpression negatively interfered with MCSF-driven mono-macrophage differentiation, as demonstrated by the reduction of the CD14+CD163+ macrophage population respect to the more immature CD14+CD163- fraction in miR-486-3p-overexpressing cells (Figure 4b and Supplementary Figure S2F). Indeed, macrophage differentiation-inducing conditions determined a homogeneous macrophage morphology in NegCTR mimic cells (Figure 4cv), while miR-486-3p mimic sample still showed a mixed monocyte/macrophage population (Figure 4cvi).

Conversely, miR-486-3p knockdown impaired the granulocyte differentiation, as demonstrated by the reduction in MPO+ and CD15+ fractions (Figure 4d) while causing an early increase in the CD14+CD163+ macrophage population (day 6, Figure 4e).

Overall, miR-486-3p overexpression and knockdown data show that miR-486-3p affects HPCs granulocyte versus mono-macrophage lineage choice, by promoting the granulocyte differentiation while constraining the macrophage differentiation. Consistently, the detection of miR-486-3p expression by quantitative real-time PCR (qRT-PCR) during granulocyte and mono-macrophage differentiation showed an early upregulation of miR-486-3p upon G-CSFdriven induction of granulocyte differentiation (Supplementary Figure S3A), while the miRNA is progressively downregulated during the mono-macrophage differentiation (Supplementary Figure S3B). 


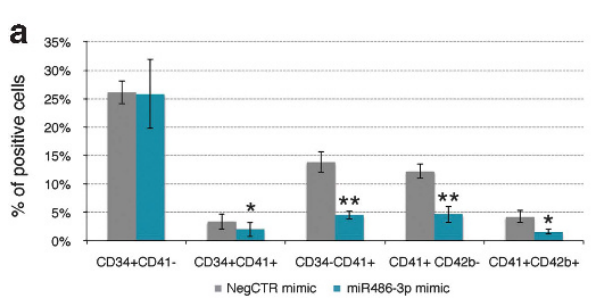

C

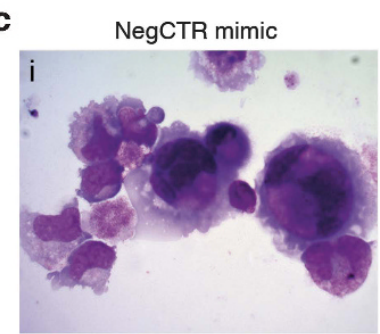

\section{d}

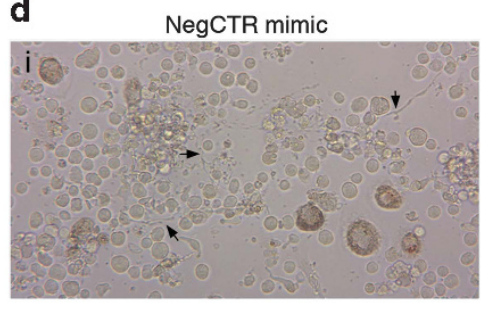

miR-486-3p mimic

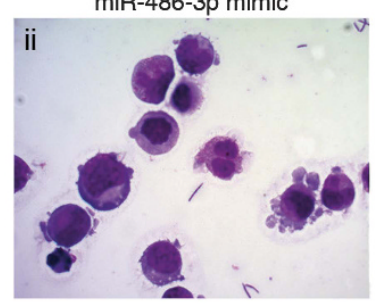

f

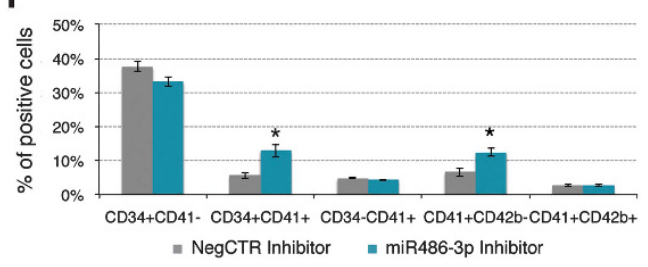

i

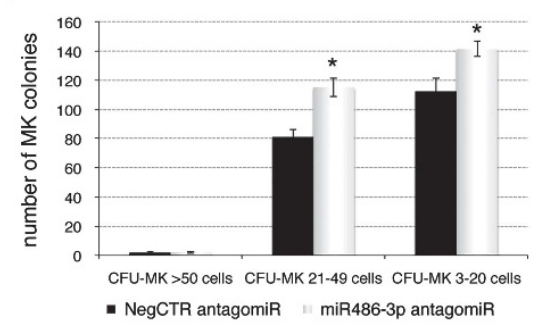

k
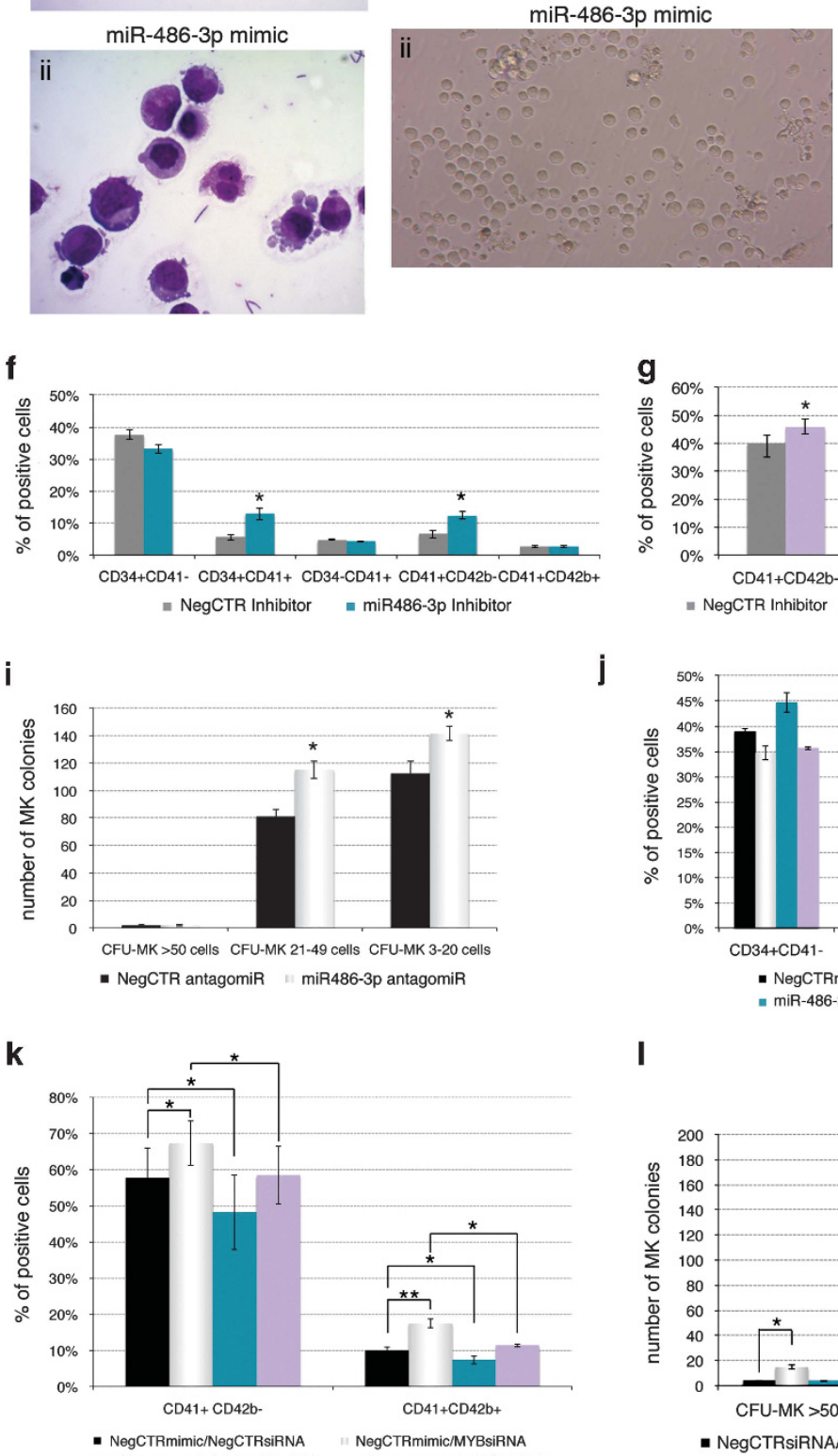

j

I b

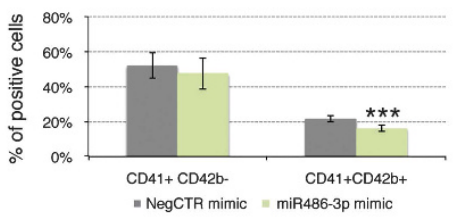

e

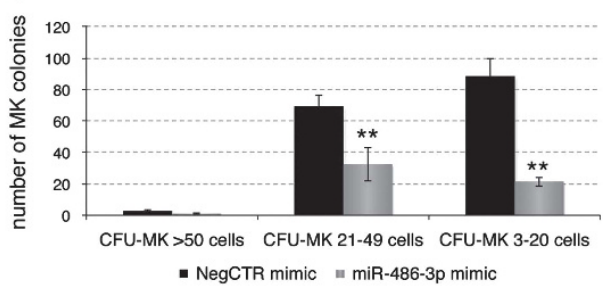

- NegCTR mimic n miR-486-3p mimic
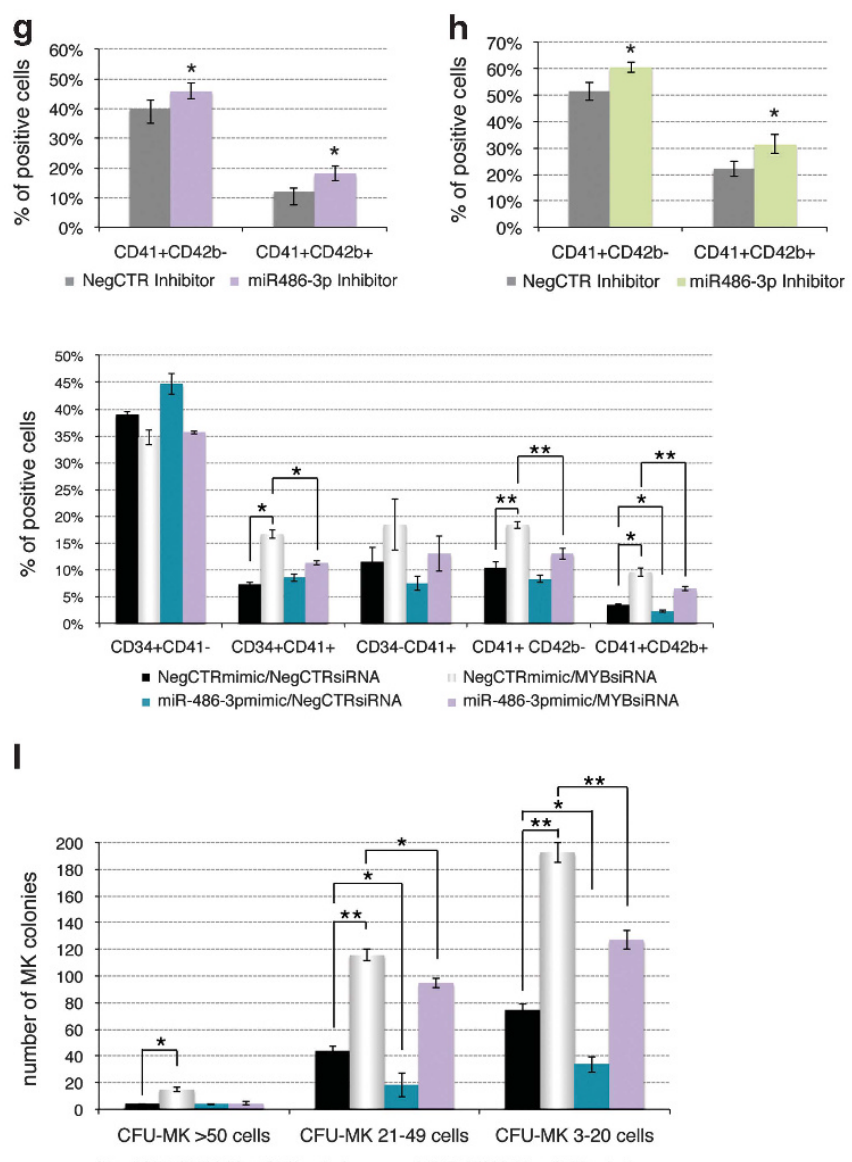

- NegCTRsiRNA/NegCTR mimic " MYBsiRNA/NegCTR mimic

- NeqCTRsiRNA/miR-486-3p mimic $=$ MYBsiRNA/miR-486-3p mimic 
Gene expression profile of miR-486-3p-overexpressing HPCs and functional validation of MAF as a miR-486-3p target. In order to unravel the molecular mechanisms underlying the effects of miR-486-3p in HPCs lineage commitment and to understand how the MYB-driven transactivation of miR-486-3p expression can contribute to explain the role of MYB in HPCs fate decision, we investigated the transcriptional changes induced by miR-486-3p overexpression in HPCs.

In a set of three independent experiments, miR-486-3p mimic and NegCTR mimic-transfected CD34+ cells were profiled by Affymetrix U219 Array (Supplementary Table S6).

Next, in order to identify the transcripts sharing a differential expression upon MYB silencing and miR-486-3p overexpression, we crossed the lists of transcripts modulated after miR-486-3p overexpression (Supplementary Table S6) and MYB silencing (Supplementary Table S2). The resulting list was represented by 18 genes with anti-correlated expression trend between miR-486-3p-overexpressing and MYB-silenced cells (Figure 5a). Among them, only the MAF (v-maf musculoaponeurotic fibrosarcoma oncogene homolog, avian, c-maf) transcript was downregulated upon miR-486-3p overexpression and displayed the putative miR-486-3p target sites in the $3^{\prime}$-UTR based on TargetScan database (http://www. targetscan.org), therefore being a predicted target for miR-486-3p. For this reason, after $q R T-P C R\left(R Q_{ \pm} S E\right.$, $0.235 \pm 0.065, P<0.01, n=4)$ and western blot (Figure $5 b$ ) validation of $M A F$ downregulation upon miR-486-3p overexpression, we tested the miR-486-3p/MAF interaction by luciferase reporter assay.

MAF gene encodes for two splicing variants, only the transcript variant 2 showing a putative miR-486-3p target site in the $3^{\prime}$ UTR (position 1380-1387 bps in $3^{\prime}$ UTR, sequence $5^{\prime}$-CUGCCCCA-3'). Figure 5 c recapitulates reporter assays results. The overexpression of miR-486-3p did not affect the luciferase activity for the samples transfected with (1) no
3'UTR (empty vector), (2) MAF variant $13^{\prime}$ UTR and (3) MAF variant $23^{\prime} U T R$, fragment $2530-4932$ bps, both lacking putative miR-486-3p-target sites. On the contrary, miR-486$3 p$ overexpression determined a reduction of luciferase activity in the sample transfected with MAF variant $23^{\prime} U T R$, fragment $1-2569 \mathrm{bps}$, by demonstrating that miR-486-3p targets MAF $3^{\prime} U T R$.

Functional validation of the MYB/miR-486-3p/MAF axis in megakaryocyte versus erythroid fate decision. At last, we unraveled the role of MAF in MYB/miR-486-3p-mediated skewing of erythroid versus megakaryocyte lineage choice. For this purpose, we firstly checked by qRT-PCR the expression of miR-486-3p and MAF during the megakaryocyte and erythroid differentiation of CD34+ cells; we detected an early downregulation of miR-486-3p (Figure 6ai) and the simultaneous upregulation of $M A F$ upon induction of the megakaryocyte differentiation (Figure 6aii). On the contrary, during the erythroid differentiation a progressive increase of miR-486-3p (Figure 6bi) and a concurrent downregulation of $M A F$ (Figure 6bii) were detected.

These data unveiled an anti-correlated expression trend for miR-486-3p and MAF during both the megakaryocyte and the erythroid differentiation of HPCs, further supporting the idea of a physiological role for miR-486-3p/MAF axis in erythroid versus megakaryocyte fate decision.

Next, we investigated whether the rescue of MAF expression could restrain the effects of miR-486-3p overexpression during the erythroid versus megakaryocyte lineage decision by concurrently overexpressing miR-486-3p and MAF in HPCs.

CD34+ cells were nucleofected with miR-486-3p or NegCTR mimics and subsequently transduced with the retroviral vector expressing the $M A F$ coding sequence (transcript variant 2, LMAFvar2IDN) or empty vector $(L X I \Delta N)$. As shown in Figure 6c, both the earlier

Figure 2 Effects of miR-486-3p overexpression, miR-486-3p knockdown and miR-486-3p rescue upon MYB silencing on megakaryocyte differentiation. (a) Flow cytometric analysis (mean \pm S.E.M.; $n=4$ ) of CD34, CD41 and CD42b expression at day 4 of megakaryocyte unilineage culture post nucleofection. (b) Flow cytometric analysis (mean \pm S.E.M.; $n=4$ ) of CD41 and CD42b expression at day 12 of megakaryocyte unilineage culture post nucleofection. (c) Morphological analysis of NegCTR mimic (i) and miR-486-3p mimic-transfected cells (ii) after May-Grünwald-Giemsa staining at day 8 of megakaryocyte unilineage culture post nucleofection in a representative experiment. Magnification, $\times 1000$. (d) Culture micrographs of NegCTR mimic (i) and miR-486-3p mimic-transfected cells (ii) at day 11 of megakaryocyte culture at $\times 200$ magnification in a representative experiment. NegCTR mimic sample (i) shows the large, polyploid cells together with several proplatelet-forming (see black arrows) cells, which are almost undetectable in miR-486-3p-overexpressing sample (ii). (e) Megakaryocyte clonogenic assay results (mean \pm S.E.M.; $n=4$ ). CFU-MKs were scored according to the manufacturer's protocol based on their size, which reflects the maturation stage of the progenitor giving rise to each colony, as large ( $>50$ cells, arising from more primitive MK progenitors), medium (21-49 cells) and small (3-21 cells, deriving from more mature megakaryocyte progenitors) colonies. The cells were plated 24 hours after the last nucleofection and scored after 12 days. Values are reported as number of megakaryocyte colonies for 4000 plated cells. ${ }^{*} P \leq 0.05,{ }^{* \star} P \leq 0.01$ and ${ }^{* \star *} P \leq 0.001$ in miR-486-3p mimic compared with NegCTR mimic sample. The efficient upregulation of miR-486-3p expression upon mimic transfection was checked at 24 hours post nucleofection by qRT$\mathrm{PCR}(\mathrm{RQ} \pm \mathrm{SE}, 74.4 \pm 19.2, P<0.05)(\mathbf{f}-\mathbf{h})$ Flow cytometric analysis (mean \pm S.E.M.; $n=3$ ) of CD34, CD41 and CD42b expression at day 4 (f) and CD41 and CD42b at day 8 (g) and day 12 (h) of megakaryocyte unilineage culture post nucleofection with miR-486-3p/NegCTR inhibitor. (i) Megakaryocyte clonogenic assay results (mean \pm S.E.M.; $n=3$ ) for miR-486-3p and NegCTR Inhibitor-transfected CD34+ cells. CFU-MKs were scored according to the manufacturer's protocol based on their size, as reported above. Values are reported as number of megakaryocyte colonies for 4000 plated cells. ${ }^{*} P \leq 0.05$ in miR-486-3p inhibitor compared with NegCTR-inhibitor sample. The efficient knockdown of miR-486-3p expression upon inhibitor transfection was checked at 24 hours post nucleofection by qRT-PCR (RQ $\pm S E, 0.593 \pm 0.161, P<0.05)(j$ and $\mathbf{k})$ Flow cytometric analysis (mean \pm S.E.M.; $n=3$ ) of CD34, CD41 and CD42b expression at day 4 (j) and CD41 and CD42b at day 12 (k) of megakaryocyte unilineage culture post nucleofection with MYBsiRNA/NegCTRsiRNA and/or miR-486-3p/NegCTR mimics. (I) Megakaryocyte clonogenic assay results (mean \pm S.E.M.; $n=3$ ) for CD34+ cells transfected with MYBsiRNA/NegCTRsiRNA and/or miR-486-3p/NegCTR mimic. CFU-MKs were scored according to the manufacturer's protocol based on their size, as reported above. Values are reported as number of megakaryocyte colonies for 4000 plated cells. ${ }^{*} P \leq 0.05,{ }^{\star *} P \leq 0.01$ and ${ }^{* \star} P \leq 0.001$. MYB and miR-486-3p expression levels were checked at 24 hours post nucleofection by qRT-PCR ( $R Q \pm S E, 0.423 \pm 0.122$ in MYBsiRNA/NegCTR mimic and $0.487 \pm 0.148$ in MYBsiRNA/miR-486-3p mimic for MYB; $75.821 \pm 13.347$ in NegCTRsiRNA/miR-486-3p mimic and $70.382 \pm 11.027$ in MYBsiRNA/miR-486-3p mimic for miR-486-3p levels; NegCTRsiRNA/NegCTR mimic set as calibrator). Abbreviations: CFU, colony forming unit; MK, megakaryocyte; MYBsiRNA, MYB-targeting siRNA; NegCTR inhibitor, negative control inhibitor; NegCTR mimic, negative control mimic; NegCTRsiRNA, negative control siRNA; and $n=$ number of experiments 


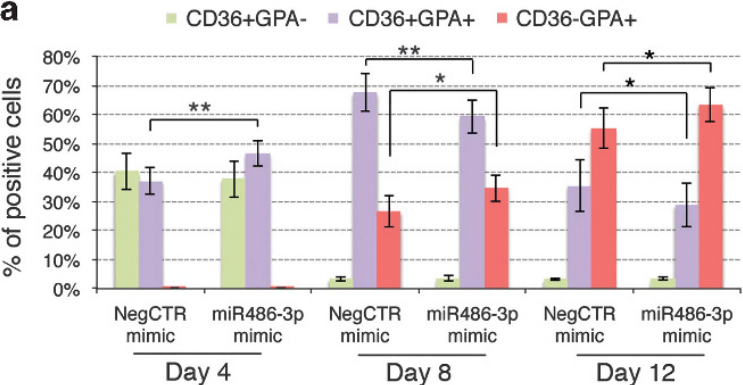

b
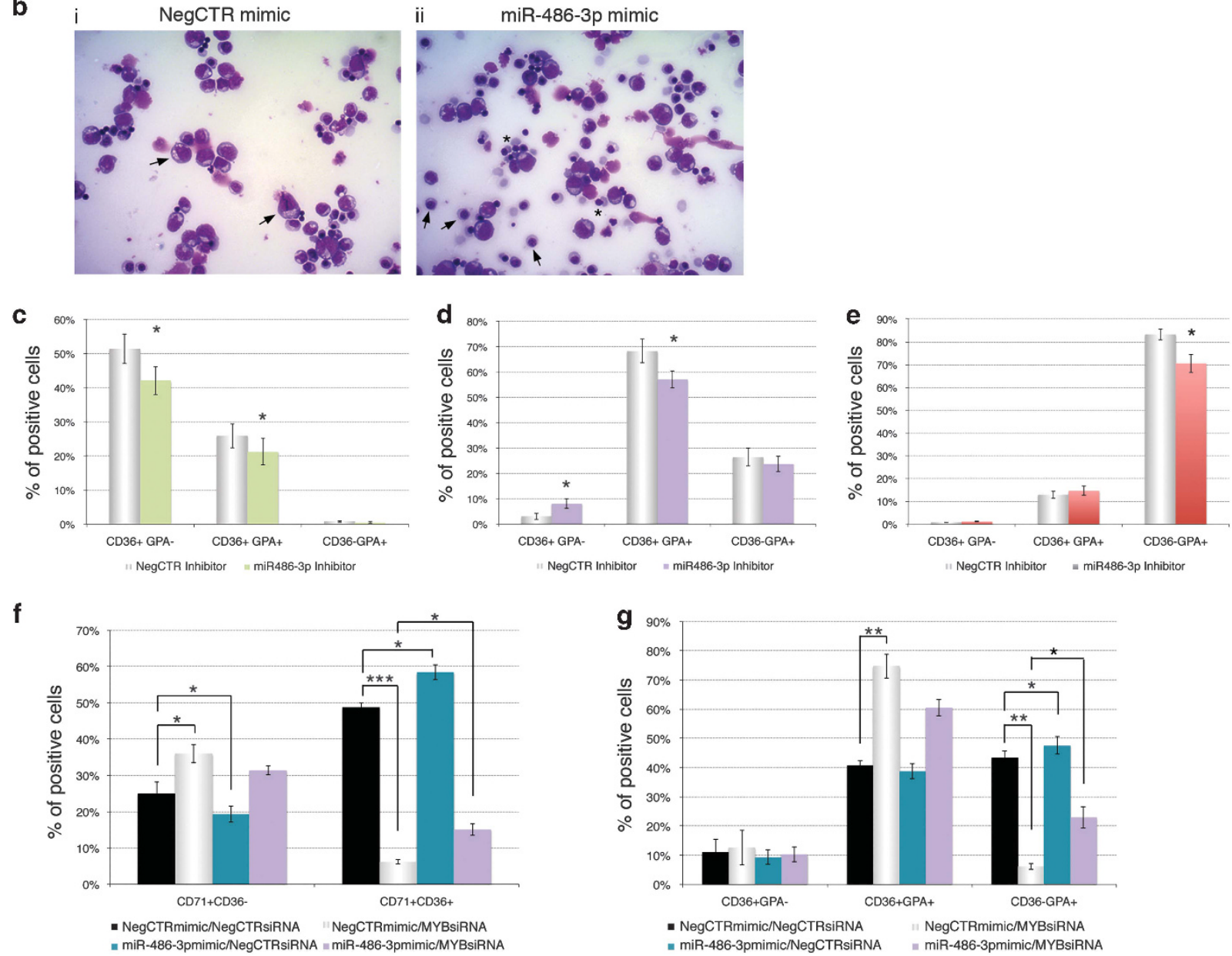

Figure 3 Effects of miR-486-3p overexpression, miR-486-3p knockdown and miR-486-3p rescue upon MYB silencing on erythroid differentiation. (a) Flow cytometric detection (mean \pm S.E.M.; $n=4$ ) of CD36 and GPA (intermediate and late erythroid markers, respectively) at days 4,8 and 12 of erythroid unilineage culture post nucleofection. ${ }^{*} P \leq 0.05$ and ${ }^{* *} P \leq 0.01$ in miR-486-3p mimic compared with NegCTR mimic sample. (b) Morphological analysis of NegCTR (i) and miR-486-3p mimic-transfected cells (ii) after May-Grünwald-Giemsa staining at day 8 of erythroid unilineage culture post nucleofection in a representative experiment. Magnification, $\times 400$. NegCTR mimic sample (i) displays more immature cells such as proerythroblasts and basophilic erythroblasts (see black arrows), while miR-486-3p mimic sample (ii) is enriched in cells from later steps of erythroid differentiation such as orthocromatic erythroblasts (see black arrows) together with nuclear extrusion phenomena (see asterisks) and enucleated reticulocytes/ erythrocytes. The efficient upregulation of miR-486-3p expression upon mimic transfection was checked at 24 hours post nucleofection by $q R T-P C R(R Q \pm S E, 74.4 \pm 19.2$, $P<.05)(\mathbf{c}-\mathbf{e})$ Flow cytometric detection (mean \pm S.E.M.; $n=3$ ) of CD36 and GPA at days 4 (c), 8 (d) and 12 (e) of erythroid unilineage culture post nucleofection with miR-486$3 p / N e g C T R$ inhibitors. ${ }^{*} P \leq 0.05$ in miR-486-3p inhibitor compared with NegCTR inhibitor sample. The efficient knockdown of miR-486-3p expression upon inhibitor transfection was checked at 24 hours post nucleofection by $q R T-P C R(R Q \pm S E, 0.593 \pm 0.161, P<.05)(\mathbf{f}, \mathbf{g})$ Flow cytometric data (mean $\pm S . E . M . ; n=3$ ) for the expression of $C D 71, C D 36$ and GPA (early, intermediate and late erythroid markers, respectively) during erythroid unilineage culture. Data reported in the graphs display the percentages of CD71+CD36(early erythroid) and CD71+CD36+ (intermediate erythroid cells) at day 4 (f) and the fractions of CD36+GPA- (intermediate), CD36+GPA+ (more mature) and CD36-GPA+ (late) erythroid cells $(\mathbf{g})$ at day 8 of erythroid unilineage culture post nucleofection with MYBsiRNA/NegCTRsiRNA and/or miR-486-3p mimic/NegCTR mimic. ${ }^{*} P \leq 0.05,{ }^{*} P \leq 0.01$ and ${ }^{* * *} P \leq 0.001$. MYB and miR-486-3p expression levels were checked at 24 hours post nucleofection by qRT-PCR $(\mathrm{RQ} \pm \mathrm{SE}, 0.423 \pm 0.122$ in MYBsiRNA/NegCTR mimic and $0.487 \pm 0.148$ in MYBsiRNA/miR-486-3p mimic for MYB; $75.821 \pm 13.347$ in NegCTRsiRNA/miR-486-3p mimic and 70.382 \pm 11.027 in MYBsiRNA/miR-486-3p mimic for miR-486-3p levels; NegCTRsiRNA/NegCTR mimic set as calibrator). Abbreviations: GPA, glycophorin A; MYBsiRNA, MYB-targeting siRNA; NegCTR mimic, negative control mimic; NegCTR inhibitor, negative control inhibitor; NegCTRsiRNA, negative control siRNA; $n=$ number of experiments 
a

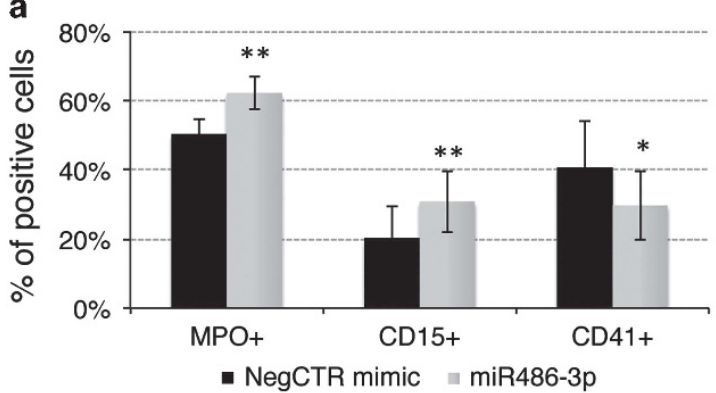

\section{b}

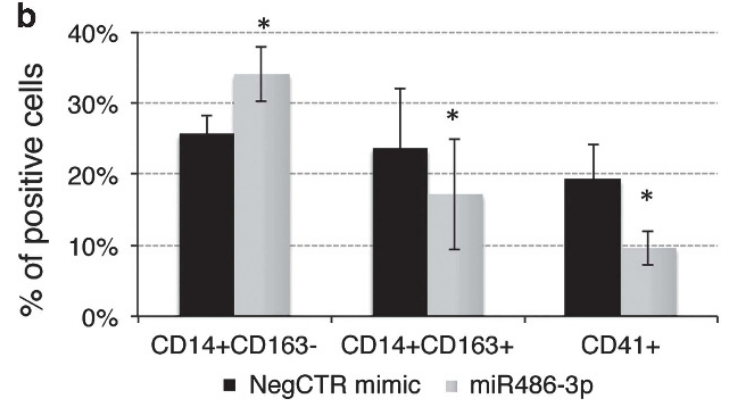

C
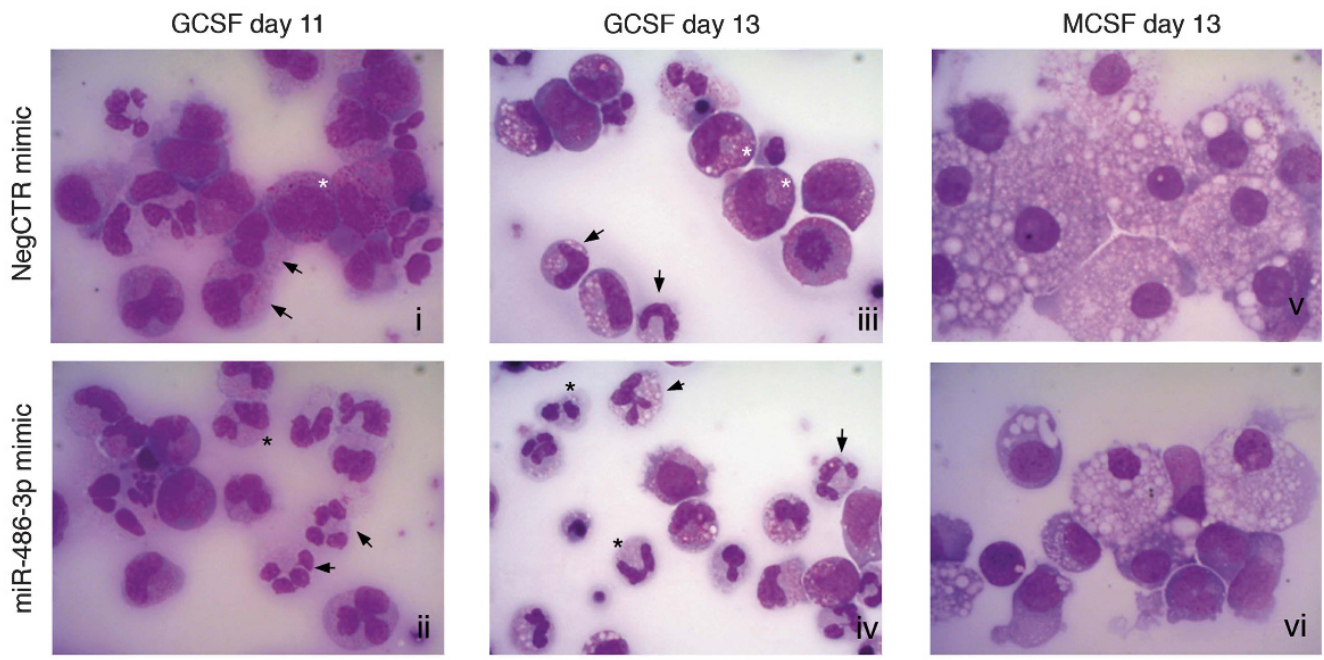

d

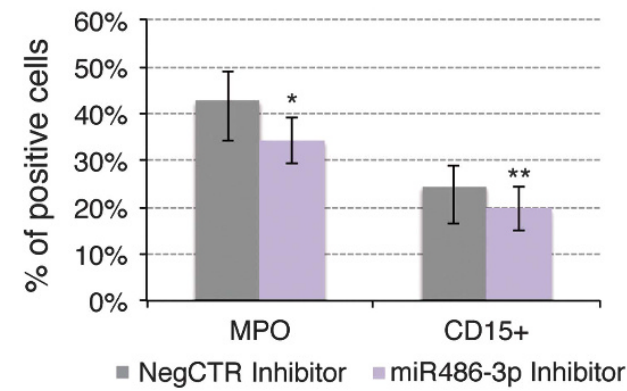

e

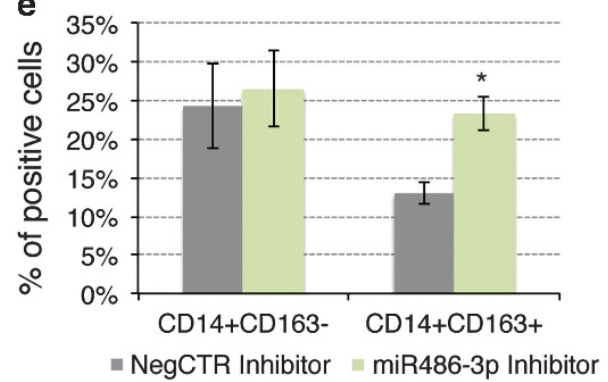

Figure 4 Effects of miR-486-3p overexpression and miR-486-3p knockdown on CD34+ cells granulocyte and mono-macrophage differentiation. (a) Flow cytometric detection (mean \pm S.E.M.; $n=4$ ) of granulocyte MPO and CD15 and megakaryocyte CD41 markers at day 10 of granulocyte unilineage culture post nucleofection. (b) Flow cytometric detection (mean \pm S.E.M.; $n=4$ ) of mono-macrophage CD14, macrophage-specific CD163 and megakaryocyte CD41 differentiation markers at day 10 of mono-macrophage unilineage culture post nucleofection. ${ }^{*} P \leq 0.05$ and ${ }^{* *} P \leq 0.01$ in miR-486-3p mimic compared with NegCTR mimic sample. (c) Morphological analysis of NegCTR (i, iii, v) and miR-486-3p mimic-transfected cells (ii, iv, vi) after May-Grünwald-Giemsa staining at days 11 (i, ii) and 13 (iii, iv) of granulocyte and day 13 (v, vi) of mono-macrophage unilineage culture post nucleofection in a representative experiment. GCSF-driven granulocyte unilineage culture highlighted at day 11 post nucleofection in NegCTR mimic sample (i) a higher fraction of more immature cells such as promyelocytes (see white asterisk) and neutrophilic metamyelocytes (black arrows), while miR-486-3p mimic sample (ii) was enriched in more mature cells, such as two-lobed (black asterisk) and segmented neutrophils (black arrows). Similarly, at 13 days of granulocyte unilineage culture NegCTR mimic sample (iii) presented a more immature phenotype, mainly represented by neutrophilic metamyelocytes (white asterisks) and neutrophilic band cells (black arrows), while miR-486-3p mimic sample (iv) was enriched in two-lobed (black asterisks) and segmented neutrophils (black arrows), representing the last steps of granulocyte differentiation. By contrast, morphological analysis at day 13 of MCSF-driven mono-macrophage unilineage culture showed a uniform macrophage morphology in NegCTR cells (v), while miR-486$3 p$-overexpressing cells (vi) still displayed a large portion of monocytes besides terminally differentiated macrophages. Magnification, $\times 1000$. The efficient upregulation of miR-486-3p expression upon mimic transfection was checked at 24 hours post nucleofection by qRT-PCR (RQ $\pm \mathrm{SE}, 74.4 \pm 19.2, P<.05)$ (d and e) Flow cytometric detection (mean \pm S.E.M.; $n=3$ ) of granulocyte MPO and CD15 at day 10 of granulocyte unilineage culture (d) and of mono-macrophage CD14 and macrophage-specific CD163 differentiation markers at day 6 of mono-macrophage unilineage culture post nucleofection (e) with miR-486-3p/NegCTR inhibitors. ${ }^{*} P \leq 0.05$ and ${ }^{* *} P \leq 0.01$ in miR-486-3p inhibitor compared with NegCTR inhibitor sample. The efficient knockdown of miR-486-3p expression upon inhibitor transfection was checked at 24 hours post nucleofection by qRT-PCR (RQ $\pm S E, 0.593 \pm 0.161, P<0.05)$. Abbreviations: GCSF, granulocyte colony stimulating factor; MCSF, macrophage colony stimulating factor; MPO, Myeloperoxidase; NegCTR mimic, negative control mimic; NegCTR inhibitor, negative control inhibitor; $n=$ number of experiments 
CD41+CD42b- population and the more mature CD41 $+\mathrm{CD} 42 \mathrm{~b}+$ fraction were increased in LMAFvar2IDN/miR$-486-3 p$ compared with LXIDN/miR-486-3p cells at 4 days of megakaryocyte unilineage culture. Similarly, the CFU-MK assay results displayed an increase in CFU-MKs in LMAFvar2IDN/miR-486-3p sample compared with LXIDN/miR-486-

a

\begin{tabular}{|c|c|c|c|c|c|c|}
\hline Probeset ID & 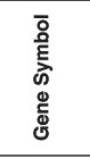 & Gene Title & 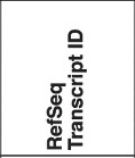 & 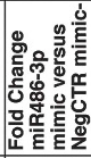 & 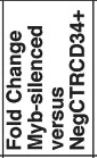 & 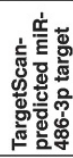 \\
\hline 11758196_s_at & IGFBP5 & insulin-like growth factor binding protein 5 & NM_000599 & 0.37 & 3.50 & - \\
\hline 11733088_at & MAF & v-maf musculoaponeurotic fibrosarcoma oncogene homolog (avian) & $\begin{array}{c}\text { NM_001031804 III } \\
\text { NM_005360 }\end{array}$ & 0.48 & 5.21 & + \\
\hline 11758577_s_at & ELOVL7 & ELOVL fatty acid elongase 7 & $\begin{array}{l}\text { NM_001104558 /III } \\
\text { NM_024930 }\end{array}$ & 0.48 & 2.12 & - \\
\hline 11753823_a_at & S100A8 & S100 calcium binding protein $A 8$ & NM_002964 & 0.49 & 3.80 & - \\
\hline 11721895_x_at & GAS1 & growth arrest-specific 1 & NM_002048 & 0.52 & 3.10 & - \\
\hline 11721894_at & GAS1 & growth arrest-specific 1 & NM_002048 & 0.54 & 2.28 & - \\
\hline 11723826_a_at & C2orf88 & chromosome 2 open reading frame 88 & \begin{tabular}{|c|} 
NM_001042519 IIII \\
NM_001042520 III \\
NM_001042521 III \\
NM_032321
\end{tabular} & 0.63 & 2.07 & - \\
\hline 11719684_a_at & NTS & neurotensin & NM_006183 & 0.65 & 4.03 & - \\
\hline 11716091_x_at & PRG2 & $\begin{array}{l}\text { proteoglycan 2, bone marrow (natural killer cell activator, eosinophil } \\
\text { granule major basic protein) }\end{array}$ & \begin{tabular}{|c|} 
NM_001243245 III \\
NM_002728 \\
\end{tabular} & 1.54 & 0.06 & - \\
\hline 11728137_at & $\mathrm{XK}$ & X-linked Kx blood group (McLeod syndrome) & NM_021083 & 1.56 & 0.34 & - \\
\hline 11757796_s_at & NCS1 & neuronal calcium sensor 1 & $\begin{array}{l}\text { NM_001128826 III } \\
\text { NM_014286 }\end{array}$ & 1.57 & 0.50 & - \\
\hline 11720400_at & NCS1 & neuronal calcium sensor 1 & $\begin{array}{l}\text { NM_001128826 III } \\
\text { NM_014286 }\end{array}$ & 1.59 & 0.49 & - \\
\hline 11716090_a_at & PRG2 & $\begin{array}{l}\text { proteoglycan 2, bone marrow (natural killer cell activator, eosinophil } \\
\text { granule major basic protein) }\end{array}$ & \begin{tabular}{|c|} 
NM_001243245 IIII \\
NM_002728
\end{tabular} & 1.59 & 0.07 & - \\
\hline 11725749_a_at & GAL & galanin prepropeptide & NM_015973 & 1.62 & 0.36 & - \\
\hline 11727280_a_at & KCNH2 & potassium voltage-gated channel, subfamily $\mathrm{H}$ (eag-related), member 2 & \begin{tabular}{|c|} 
NM_000238 IIII \\
NM_001204798 III \\
NM_172056 III \\
NM_172057
\end{tabular} & 1.63 & 0.06 & - \\
\hline 11718479_x_at & STAR & steroidogenic acute regulatory protein & $\begin{array}{l}\text { NM_000349 } / I I I \\
\text { NM_001007243 }\end{array}$ & 1.65 & 0.42 & - \\
\hline 11720401_at & NCS1 & neuronal calcium sensor 1 & $\begin{array}{l}\text { NM_001128826 III } \\
\text { NM_014286 }\end{array}$ & 1.80 & 0.42 & - \\
\hline 11733662_a_at & KCNH2 & potassium voltage-gated channel, subfamily $\mathrm{H}$ (eag-related), member 2 & \begin{tabular}{|c|} 
NM_000238 III \\
NM_001204798 III \\
NM_172056 IIII \\
NM_172057 \\
\end{tabular} & 1.83 & 0.03 & - \\
\hline
\end{tabular}

b

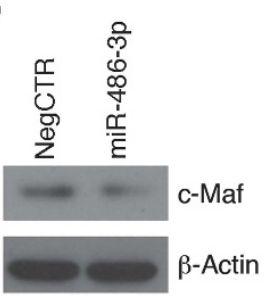

c

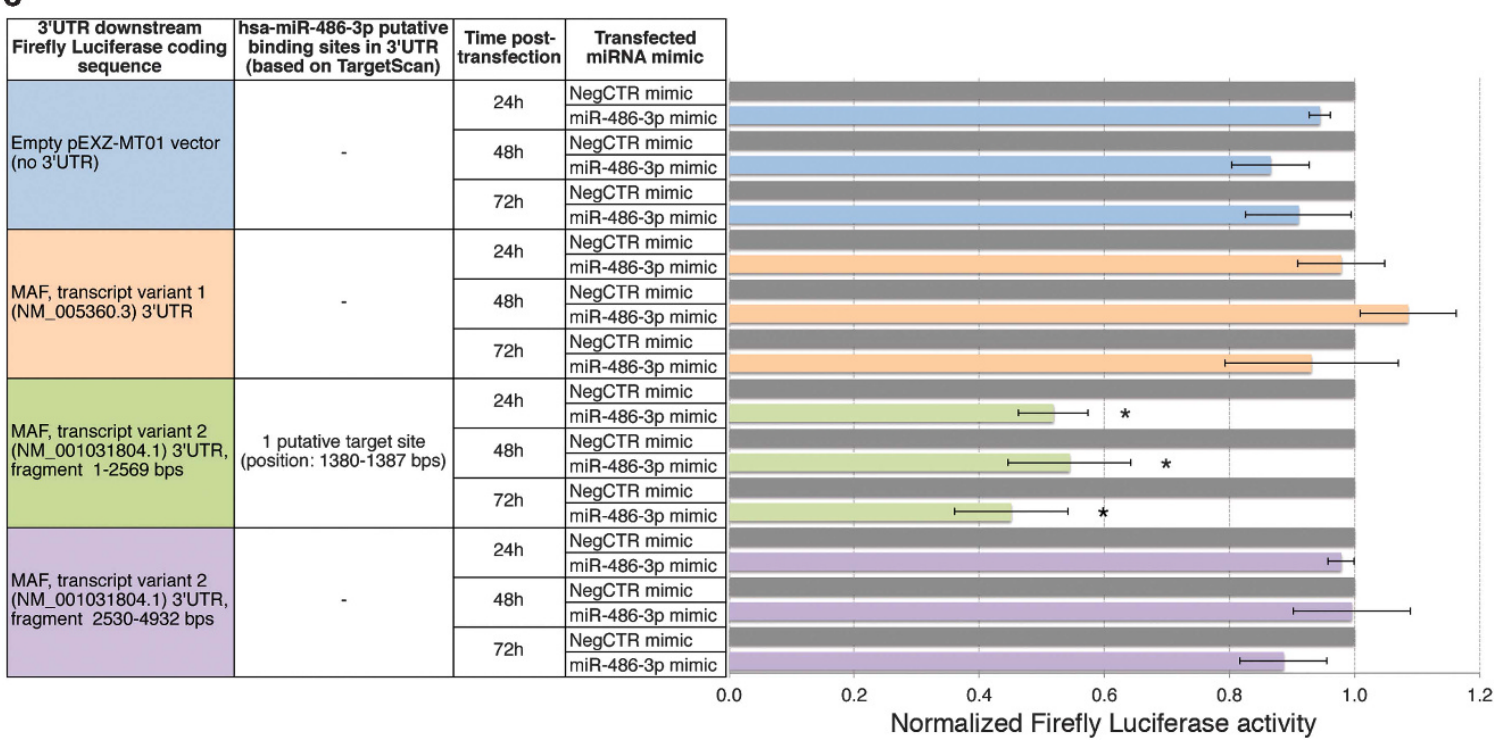


$3 p$ cells (Figure $6 d$ ). On the contrary, the erythroid CD36 +GPA- and CD36+GPA+ fractions were reduced in LMAFvar2IDN/miR-486-3p cells compared with LXIDN/miR-486-3p cells (Figure $6 e$ ). Collectively these data demonstrate that the constitutive expression of MAF can constrain the effects of miR-486-3p overexpression during the erythroid versus megakaryocyte lineage choice.

Finally, in order to check whether the upregulation of MAF could be relevant in the skewing of erythroid versus megakaryocyte fate decision upon MYB silencing, we studied the effects of the siRNA-mediated silencing of MYB (MYBsiRNA; Supplementary Table S1), MAF (MAFsiRNA; Supplementary Table S1) or both (MYBsiRNA+MAFsiRNA) in CD34+ cells.

As shown in Figure 6f, the expansion of the megakaryocyte lineage upon MYB silencing (MYBsiRNA) was restrained by the co-silencing of MAF (MYBsiRNA+MAFsiRNA), as demonstrated by the reduction in CD41+ population (Figure 6f, see also the CD41+CD42b- and CD41+CD42b+ populations at 8 and 12 days post nucleofection, respectively, Supplementary Figures S4A and B).

In line with these data, the CFU-MK assay results (Figure 6g, Supplementary Figure S4C) demonstrated that the abnormal expansion of megakaryopoiesis in MYBsiRNA sample was significantly constrained by the co-silencing of MAF (MYBsiRNA+MAFsiRNA).

Similarly, the effects of the MYB/MAF co-silencing in erythropoiesis were studied. Flow cytometric detection of erythroid differentiation markers demonstrated that, whereas MYB silencing caused a CD71+CD36-/CD71+CD36+ erythroid transition arrest, the co-silencing of MAF was able to partially overcome this differentiation block at 4 days of erythroid unilineage culture (Figure 6h, MYBsiRNA+MAFsiRNA compared with MYBsiRNA), by restoring the CD71+ CD36+ and CD36+GPA- fractions (Figures 6h and i, MYBsiRNA+MAFsiRNA compared with MYBsiRNA).

Collectively, these data demonstrate that MAF silencing in HPCs is able to reverse at least in part the effects of MYB silencing on erythroid versus megakaryocyte lineage choice.

\section{Discussion}

In order to shed some light on the MYB-driven miRNAmediated regulation of gene expression during HPCs lineage commitment, in this study we profiled the mRNA and miRNA expression changes induced by MYB silencing in human CD34+ cells. The integrative analysis of miEP and GEP data allowed us to identify 242 miRNA-mRNA pairs differentially expressed in MYB-silenced compared with the control CD34+ cells and with the anti-correlated expression trend. Among the miRNAs with the highest number of putative target mRNAs, we further investigated miR-486-3p as strongly downregulated upon MYB silencing together with the host gene ankyrin-1 (ANK1). ${ }^{18,19}$ After showing that MYB controls miR-486-3p expression by driving ANK1 transcription, we investigated the role of miR-486-3p on lineage commitment through overexpression and knockdown experiments in CD34+ cells.

We demonstrate that miR-486-3p supports erythroid and granulocyte differentiation, while negatively interferes with megakaryocyte and macrophage differentiation. Indeed, miR-486-3p overexpression restrained the megakaryocyte differentiation and enhanced the erythroid differentiation. Conversely, miR-486-3p knockdown impaired the erythroid differentiation while favouring megakaryocyte differentiation. Noteworthy, the rescue of miR-486-3p expression was able to partially constrain the skewing of erythroid versus megakaryocyte fate decision detected upon MYB silencing in HPCs, further confirming the functional relevance of $\mathrm{miR}-486-3 p$ in MYB-dependent control of hematopoiesis.

Next, in order to unravel the molecular mechanisms by which miR-486-3p affects the lineage commitment and differentiation of HPCs, we profiled the gene expression changes upon miR-486-3p overexpression. In this way, we identified MAF as a computationally predicted miR-486-3p target downregulated after miR-486-3p-overexpression and upregulated upon MYB silencing. Indeed, 3'UTR reporter assays provided the experimental evidence that MAF is a target of miR-486-3p.

Interestingly, miR-486-3p and MAF display an anticorrelated expression trend during both erythroid and megakaryocyte differentiation, further stressing the role of the miR-486-3p/MAF axis in erythroid versus megakaryocyte lineage choice. In order to definitively clarify the functional role of the MYB/miR-486-3p/MAF axis, we demonstrated that the rescue of MAF expression can restrain the effects of miR-486-3p overexpression in erythroid and megakaryocyte commitment. In addition, in MYB-silenced HPCs the co-silencing of MAF partially reversed the skewing of erythroid versus megakaryocyte lineage choice, by restraining the expansion of megakaryocyte lineage and partially rescuing the impairment of erythropoiesis.

As a whole, our data identify the MYB/miR-486-3p/MAF axis as a new molecular mechanism through which MYB favors erythropoiesis while restraining megakaryopoiesis.

The miR-486-3p overexpression and knockdown data also demonstrated that miR-486-3p promotes granulocyte differentiation while repressing mono-macrophage differentiation.

\footnotetext{
Figure 5 Gene expression profiling in miR-486-3p-overexpressing CD34+ cells and validation of the miR-486-3p/MAF 3'UTR interaction. (a) DEGs in miR-486-3poverexpressing compared with the NegCTR mimic-transfected CD34+ cells at 24 hours post nucleofection. DEGs in table are selected as modulated in both miR-486-3poverexpressing compared with NegCTR mimic cells and in MYB-silenced compared with NegCTRsiRNA cells. For both these comparisons, transcripts modulations are reported as fold change. Transcripts in the table are ranked based on the fold change in miR-486-3p mimic compared with NegCTR mimic samples. Data for the computational prediction of miR-486-3p targets from TargetScan are reported in the last column. (b) Western blotting analysis of MAF protein levels in protein lysates from miR-486-3p mimic-transfected compared with NegCTR mimic-transfected CD34+ cells at 24 hours after the last of two nucleofection cycles (i.e. at the same time point selected for gene expression profiling). $\beta$-actin protein levels are reported as loading control. (c) Renilla-normalized Firefly luciferase activity in K562 cells nucleofected with either a NegCTR mimic or a miR-486-3p mimic and the indicated $3^{\prime}$ untranslated region ( $3^{\prime}$ UTR) luciferase reporter vectors. Renilla-normalized Firefly luciferase activity (mean \pm S.E.M.; $n=3$ ) was measured at 24,48 and 72 hours post nucleofection. Each bar represents the luciferase activity upon miR-486-3p overexpression normalized to the value of the same $3^{\prime} U T R$ luciferase vector upon NegCTR mimic transfection. ${ }^{*} P<0.05$ compared with NegCTR mimic sample.
} 
a
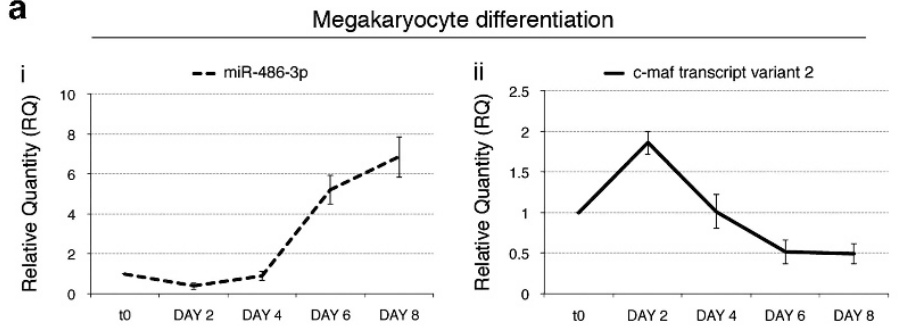

b
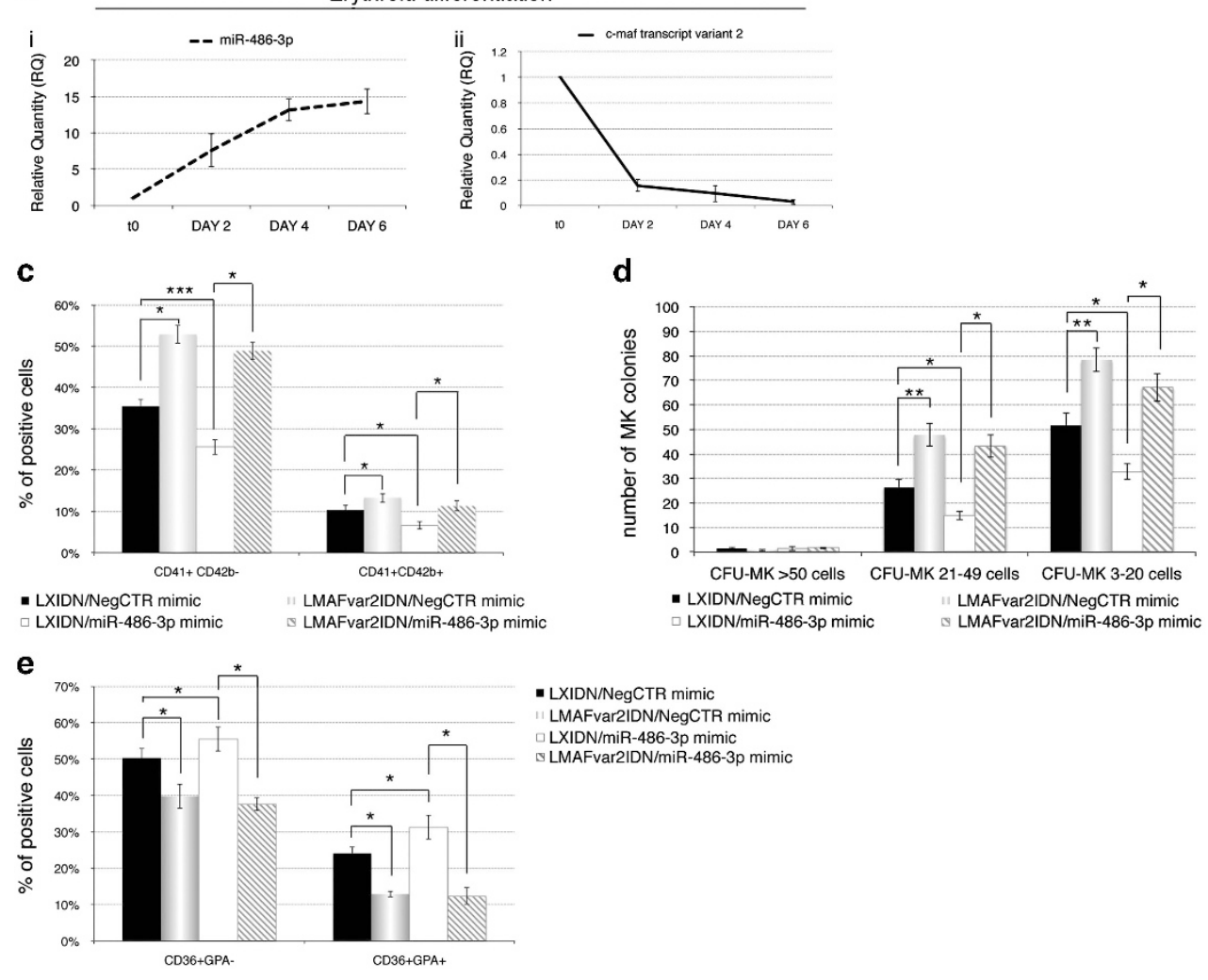

f - NegCtRsiRnA $=$ MAFsiRnA $\square$ MYBsiRnA $\square$ MAFsiRnA + MYR:

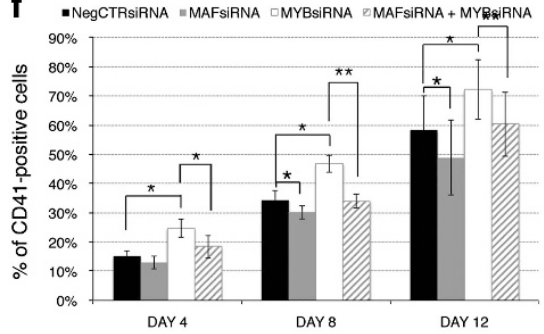

- LXIDN/NegCTR mimic

LMAFvar2IDN/NegCTR mimic

LXIDN/miR-486-3p mimic

LMAFvar2IDN/miR-486-3p mimic

h. NegCTR siRNA =MAFsiRNA $\square$ MYBsiRNA $\triangle M Y B s i R N A+M A F s i R N A$

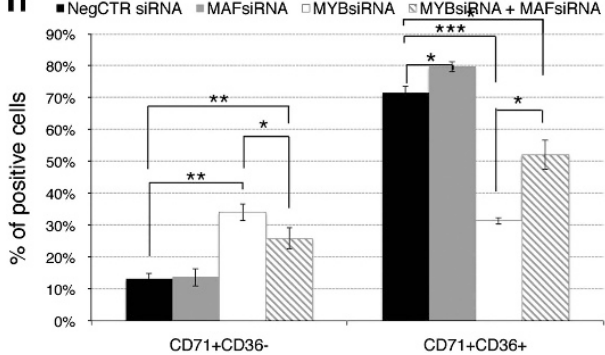

g
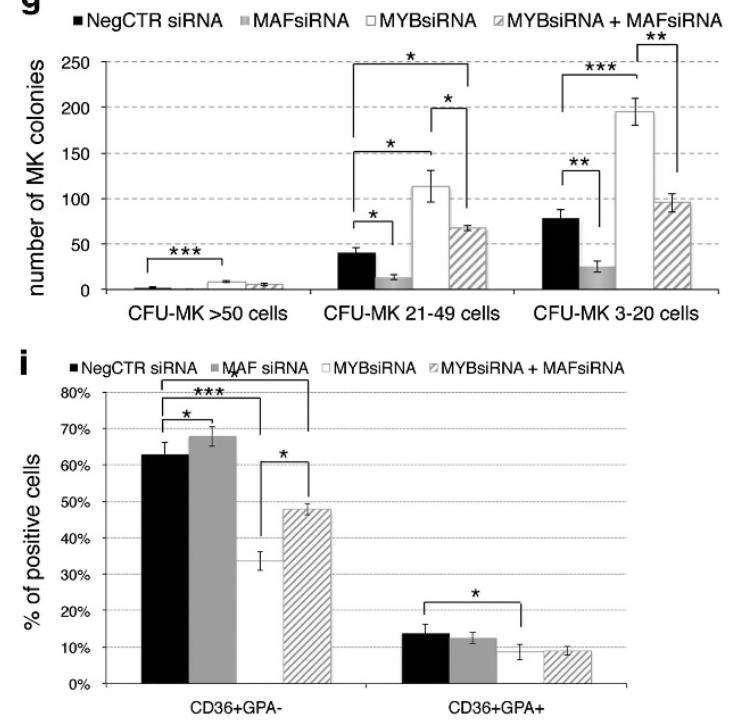
These data, together with the anti-correlated expression levels detected for miR-486-3p and $M A F$ in the earlier phases of granulocyte and mono-macrophage differentiation, strongly suggest a role for MYB/miR-486-3p/MAF axis even in granulocyte versus mono-macrophage lineage commitment. As shown by Hegde et al., ${ }^{11}$ the overexpression of MAF in myeloid cells favors the mono-macrophage differentiation by a MAF/MYB physical interaction negatively interfering with MYB activity. Therefore, the MYB-driven induction of miR-486-3p and the subsequent downregulation of $M A F$ during the granulocyte lineage commitment could be pivotal to elicit the MYB-dependent transcriptional control of granulocyte lineage priming.

Noteworthy, in agreement with Hegde et al., ${ }^{11}$ we detected a remarkable induction of MAF expression during monomacrophage differentiation (Supplementary Figure S3), also suggesting that other mechanisms besides the MYB/miR-486$3 p / M A F$ axis should be inferred to explain the strong induction of MAF expression during this process.

Respect to the aforementioned inhibitory MYB/MAF interaction ${ }^{11,22,23}$ our data add new insights on the reciprocal regulation of these transcription factors during hematopoietic lineage commitment and differentiation, by demonstrating that MYB down-regulates MAF expression levels by inducing miR-486-3p expression.

To elucidate the molecular mechanisms of erythroid versus megakaryocyte HPCs fate decision involving MYB, an increasing number of studies identified miRNAs that affect hematopoiesis by targeting MYB. ${ }^{24-28}$ However, no data dealing with the MYB-driven transcriptional control of miRNAs expression and the relevance of this molecular mechanism in HPCs lineage choice were available till now.

In this study, for the first time we shed some light on the transcriptional control of miRNA expression as a further mechanism by which MYB regulates hematopoiesis. Of note, GEP/miEP integrative analysis data allowed us to demonstrate that MYB affects HPCs fate decision through the transcriptional control of miR-486-3p expression.

In line with our data, both miR-486-3p and miR-486-5p, generated from the processing of a common miR-486 precursor, were recently reported to be upregulated during normal erythropoiesis $^{20,29}$ and in myeloid leukemogenesis. ${ }^{17,20,29}$ The demonstration that miR-486-5p, besides miR-486-3p, affects the erythroid differentiation further emphasizes the importance of $\mathrm{miR}-486-3 \mathrm{p} / \mathrm{miR}-486-5 \mathrm{p}$ induction in MYBdependent control of erythropoiesis.

As a whole, here we uncover and characterize the MYB/miR-486-3p/MAF axis (Figure 7) as a new mechanism underlying MYB-driven control of erythroid versus megakaryocyte lineage choice.

\section{Materials and Methods}

Ethics statement. Human CD34+ cells were purified upon donor's informed written consent from umbilical CB samples, collected after normal deliveries, according to the institutional guidelines for discarded material (Clearance of Ethical Commitee for Human experimentation of Modena: Secretary office Saverio Santachiara, santachiara.saverio@policlinico.mo.it, approval date: 18.01.2005; approval file number \# 793/CE).

Human CD34+ hematopoietic progenitor cells purification. Umbilical CB samples were collected after normal deliveries, according to the institutional guidelines for discarded material. CB CD34+ cells were purified as previously described. ${ }^{3}$ After immunomagnetic separation, CD34+ cells were seeded in 24-well plates at $5 \times 10^{5} / \mathrm{ml}$ in Iscove's modified Dulbecco's medium (IMDM) (GIBCO, Grand Island, NY, USA) containing 20\% human serum (Bio-Whittaker, Walkersville, MD, USA), stem cell factor (SCF) $(50 \mathrm{ng} / \mathrm{ml})$, Flt3-ligand (FLT3L) $(50 \mathrm{ng} / \mathrm{ml})$, thrombopoietin (THPO) $(20 \mathrm{ng} / \mathrm{ml})$, interleukin-6 (IL6) $(10 \mathrm{ng} / \mathrm{ml})$ and interleukin-3 (IL3) $(10 \mathrm{ng} / \mathrm{ml})$ (all from Miltenyi Biotec, Auburn, CA, USA) and electroporated 24 hours later.

Nucleofection of CD34+ cells. Human CD34+ cells were transfected by using the 4D-Nucleofector System (Lonza Group Ltd, Basel, Switzerland) as previously reported. ${ }^{17}$ Briefly, starting from the day after CD34+ cell purification, each sample was electroporated three times, once every 24 hours, with a mix of three small interfering RNAs (siRNAs) targeting human MYB and/or MAF mRNAs (Supplementary Table S1) (Life Technologies, Carlsbad, CA, USA). For each electroporation, $4 \times 10^{5} \mathrm{CD} 34+$ cells were resuspended in $100 \mu$ l of P3 Primary Cell Solution (Lonza Group Ltd), containing $3 \mu \mathrm{g}$ of siRNA mix, and pulsed with the program DS112. To exclude non-specific effects caused by interfering RNA (RNAi) nucleofection, one sample transfected with a non-targeting siRNA (NegCTRsiRNA; Life Technologies) for each experiment was included.

Similarly, miRNA mimics and inhibitors transfection was performed as previously reported. ${ }^{17}$ Briefly, CD34+ cells were nucleofected twice, once every $24 \mathrm{~h}$, with $3 \mu \mathrm{g}$ of mirVana miR-486-3p mimic or mirVana miRNA mimic negative control (NegCTR

Figure 6 Rescue of MAF expression in miR-486-3p-overexpressing cells and co-silencing of MYB and MAF in CD34+ cells. (a and $\mathbf{b}$ ) Expression kinetics of miR-486-3p (ai, bi) and MAFmRNA (transcript variant 2) (aii, bii) during the megakaryocyte (a) and the erythroid (b) differentiation of CD34+ cells; qRT-PCR-detected modulations are reported as $\mathrm{RQ}$ compared with $\mathrm{CD} 34+$ cells (day 0). Values in the graphs are reported as mean \pm S.E.M. $(n=3)$. (c) Flow cytometric analysis $($ mean \pm S.E.M.; $n=3$ ) of CD41 and CD42b expression at day 4 of megakaryocyte unilineage culture post purification of miR-486-3p/NegCTR mimic-transfected, LMAFvar2IDN/LXIDN-transduced cells. (d) Megakaryocyte clonogenic assay results (mean \pm S.E.M.; $n=3$ ) for miR-486-3p/NegCTR mimic-transfected, LMAFvar2IDN/LXIDN-transduced CD34+ cells. Cells were plated after the NGFRbased selection of transduced cells and scored after 12 days. CFU-MKs were scored according to the manufacturer's protocol based on their size, as reported in Figure 2 legend. Values are reported as number of megakaryocyte colonies for 10000 plated cells. ${ }^{*} P \leq 0.05,{ }^{* \star} P \leq 0.01$ and ${ }^{* * *} P \leq 0.001$. (e) Flow cytometric detection (mean $\pm S$.E.M.; $n=3$ ) of CD36+GPA- and CD36+GPA + erythroid populations at day 4 of erythroid unilineage culture post purification of miR-486-3p/NegCTR mimic-transfected, LMAFvar2IDN/LXIDNtransduced cells. In NGFR+ cells the efficient upregulation of miR-486-3p expression was checked by qRT-PCR (RQ \pm SE, 58.3 \pm 13.2 in miR-486-3p mimic/LXIDN and $67.9 \pm 17.1$ in miR-486-3p mimic/LMAFvar2IDN, NegCTR mimic/LXIDN sample set as calibrator), while MAF protein levels were detected by western blot (data not shown). (f) Flow cytometric analysis (mean \pm S.E.M.; $n=4$ ) of the CD41 expression at day 4, 8 and 12 of megakaryocyte culture post nucleofection. (g) Megakaryocyte clonogenic assay results (mean \pm S.E.M.; $n=4$ ). Cells were plated 24 hours after the last nucleofection and scored after 12 days. CFU-MKs were scored according to the manufacturer's protocol based on their size, as detailed in Figure 2 legend. Values are reported as number of megakaryocyte colonies for 4000 plated cells. (h and i) Flow cytometry data (mean \pm S.E.M.; $n=4$ ) for the expression of of CD71, CD36 and GPA (early, intermediate and late erythroid markers, respectively) during erythroid unilineage culture. Data reported in the graphs display the percentages of CD71+CD36- (early) and CD71+CD36+ (intermediate) erythroid cells (h), besides the fractions of CD36+GPA- (intermediate) and CD36+GPA+ (more mature) cells (i) at day 4 of erythroid unilineage culture post nucleofection. qRT-PCR data demonstrated that, as expected, MAF was upregulated upon MYB silencing (RQ \pm SE, $6.758 \pm 0.559, P<0.01$ in MYBsiRNA compared with NegCTRsiRNA, $n=4)$; on the contrary it was efficiently downregulated after MAF silencing in both MAFsiRNA compared with NegCTRsiRNA (RQ \pm SE, $0.329 \pm 0.035, P<0.01, n=4)$ and MYBsiRNA+MAFsiRNA compared with MYBsiRNA $(R Q \pm S E, 2.055 \pm 0.213, P<0.05, n=4)$ at 24 hours post nucleofection. ${ }^{*} P \leq 0.05,{ }^{* *} P \leq 0.01$ and ${ }^{* *} P \leq 0.001$. Abbreviations: CFU, colony forming unit; GPA, glycophorin A; MK, megakaryocyte; NegCTRsiRNA, negative control siRNA; $n=$ number of experiments 
mimic, both from Life Technologies), by using the above mentioned electroporation protocol DS112.

Similarly, CD34+ cells were nucleofected four times, once every 24 hours, with $3 \mu \mathrm{g}$ of miR-486-3p miRCURY LNA Power Inhibitor or miRCURY LNA Power Inhibitor Negative Control A, by using the electroporation protocol DS112.

At last, for the experiments of miR-486-3p rescue in MYB-silenced cells, CD34+ cells were nucleofected three times, once every $24 \mathrm{~h}$, with $3 \mu \mathrm{g}$ of mirVana miR-4863p/NegCTR mimic and $3 \mu \mathrm{g}$ of MYB-targeting/NegCTRsiRNA.

RNA extraction. Total cellular RNA, including small RNAs, was isolated from $3 \times 10^{5}$ cells for each sample using the miRNeasy MicroRNA isolation kit (QIAGEN) following the manufacturer's recommendations. RNA samples concentration and purity (assessed as $260 / 280 \mathrm{~nm}$ and $260 / 230 \mathrm{~nm}$ ratios) were evaluated by NanoDrop ND-1000 spectrophotometer (NanoDrop Technologies; Wilmington, DE, USA), while RNA integrity was assessed by using the Agilent 2100 Bioanalyzer (Agilent Technologies; Waldbrunn, Germany).

Gene expression profiling (GEP). GEP was performed on RNA samples isolated from MYB-silenced (MYBsiRNA sample) and non-targeting negative control siRNA-transfected (NegCTRsiRNA sample) CD34+ cells at $24 \mathrm{~h}$ after 1, 2 and 3 nucleofections from five independent experiments. miEP was performed on the same RNA samples isolated from CD34+ cells at 24 hours after three nucleofections and used for GEP $(n=5)$.

GEP cDNA synthesis and biotin-labeled target synthesis were performed using the GeneAtlas $3^{\prime}$ IVT Express Kit according to the protocol supplied by Affymetrix. The HG-U219 Array Strips (Affymetrix) hybridization, staining and scanning were performed by using the GeneAtlas Platform.

Differentially expressed genes (DEGs) were selected on robust multiarray average (RMA)-normalized data through a supervised analysis using the ANOVA module supplied by the Partek GS. 6.6 Software Package (http://www.partek.com). In particular, we selected all the probesets with a fold change contrast $\geq 2$ for DEGs in the pairwise comparison between MYBsiRNA and NegCTRsiRNA samples and a false discovery rate (FDR) ( $q$-value) $<0.05$.

In a similar way, GEP was performed on RNA samples isolated from miR486-3p mimic and NegCTR mimic CD34+ cells at 24 hours after the last nucleofection from three independent experiments. GEP data were analyzed by using the Partek GS 6.6 Software Package as reported above, by selecting the probesets with a fold change contrast $\geq 1.5(P<.05)$ for DEGs in the pairwise comparison between miR486-3p mimic and NegCTR mimic samples.

All the GEP data have been deposited in the NCBI's Gene Expression (GEO) public repository ${ }^{30}$ (http://www.ncbi.nlm.nih.gov/geo; series GSE60301, subseries GSE60299 for GEP in MYB-silenced CD34+ cells and GSE60298 for miR-486-3p overexpressing CD34+ cells).

miRNA expression profiling (miEP). miEP was performed by using the miRCURY LNA Universal RT microRNA PCR system, ready-to-use human miRNome panels I and II (Exiqon) according to the manufacturer's instructions.

For each sample, $40 \mathrm{ng}$ of total RNA were reverse transcribed in $40 \mu$ reactions. Next, CDNA was diluted 200 -fold and assayed in $10 \mu \mathrm{l}-\mathrm{PCR}$ reactions according to the protocol for miRCURY LNA Universal RT microRNA PCR protocol; each miRNA was assayed once by qPCR on the miRNA ready-to-use PCR, Human panel I and panel II.

RT-qPCR was performed in a LightCycler 480 Real-Time PCR System (Roche, Indianapolis, IN, USA) in the 384-well format. The Roche LightCycler software was used in order to analyze amplification curves in order to determine threshold cycle ( $\mathrm{Ct}$, by the second derivative method) and to analyze melting curve for each well.

RT-qPCR data were analyzed using the Exiqon GenEx software for the inter-plate calibration, the definition of a $\mathrm{Ct}=40$ as detection cutoff, the labeling of not-amplified wells as missing data and the setting of a cutoff for miRNAs with a low call-rate (i.e. the deletion of the assays with a detection rate $<30 \%$ of samples, therefore keeping the assays detected in at least 3 out of 10 analyzed samples for further analyses).

For RT-qPCR data normalization, the comparative cycle threshold (CT) method was used by setting SNORD49A as reference gene, as the best normalizer identified among the candidates by the NormFinder algorithm. ${ }^{31}$ The $R Q$ value was expressed as $2^{-\Delta \Delta C T}$.

Statistical analysis on $\Delta$ CT values between MYBsiRNA and NegCTRsiRNA sample (set as calibrator) was performed by a two-tail paired $t$-Student test by using the GenEx software. Differentially expressed miRNAs (DEMs) were selected as the
miRNAs with a relative quantity $(R Q) \geq 1.5$ or $\leq 0.67(P<.05)$ in the pairwise comparison between MYBsiRNA and NegCTRsiRNA samples.

The miEP data for MYB silencing experiments in CD34+ cells have been deposited in the GEO public database ${ }^{30}$ together with the GEP data from the same samples (http://www.ncbi.nlm.nih.gov/geo; series GSE60301, subseries 60300 for miEP data).

GEP and miEP integrative analysis. Ingenuity Pathway Analysis software (IPA, version 8.6; Ingenuity Systems; Redwood City, CA, USA http://www.ingenuity. com) was used to generate miRNA-mRNA interactions between the DEMs and their putative target differentially expressed genes (DEGs) with anti-correlated expression upon MYB silencing in CD34+ cells at 24 hours after the last nucleofection. IPA combines computationally predicted targets with gene expression data. Through the microRNA Target Filter, IPA software enables the identification of the DEMs putative targets within the DEG list according to at least one out of four different databases (TargetScan, miRecords, Tarbase or Ingenuity expert findings).

miR-486-3p and MAF kinetics during erythroid, megakaryocyte, granulocyte and mono-macrophage differentiation. After immunomagnetic separation, CD34+ cells were seeded in 24-well plates at a density of $5 \times 10^{5} / \mathrm{ml}$ in serum-free medium SYN-H (ABCell-Bio, Paris, France) supplemented with SCF $(10 \mathrm{ng} / \mathrm{ml}), I L-3(2 \mathrm{ng} / \mathrm{ml})$ and IL-6 $(1 \mathrm{ng} / \mathrm{ml})$. After a first phase of expansion, at 6 days of culture the cells were seeded $\left(5 \times 10^{5} / \mathrm{ml}\right)$ in IMDM added with $20 \%$ BIT serum substitute (bovine serum albumin, insulin, and transferrin; StemCell Technologies, Vancouver, BC, Canada), SCF (10 ng/ml), IL-3 (2 ng/ml), IL-6 $(1 \mathrm{ng} / \mathrm{ml})$ and EPO $(4 \mathrm{U} / \mathrm{ml})$ to induce erythroid differentiation. The medium was replaced every 3 days. miR-486-3p, MAF and ANK1 expression levels were detected by qRT-PCR at different time points (i.e. days $0,2,4$ and 6 ) after the seeding of cells in erythroid differentiation-inducing culture conditions.

In a similar way, after immunomagnetic sorting, CD34+ cells were seeded in 24well plates at a density of $5 \times 10^{5} / \mathrm{ml}$ in serum-free medium SYN-H (ABCell-Bio, Paris, France) supplemented with SCF (50 ng/ml), FLT3L (50 ng/ml), THPO (20 ng/ml), IL-3 $(10 \mathrm{ng} / \mathrm{ml})$ and IL-6 $(10 \mathrm{ng} / \mathrm{ml})$. After a first phase of expansion, at 4 days of culture the
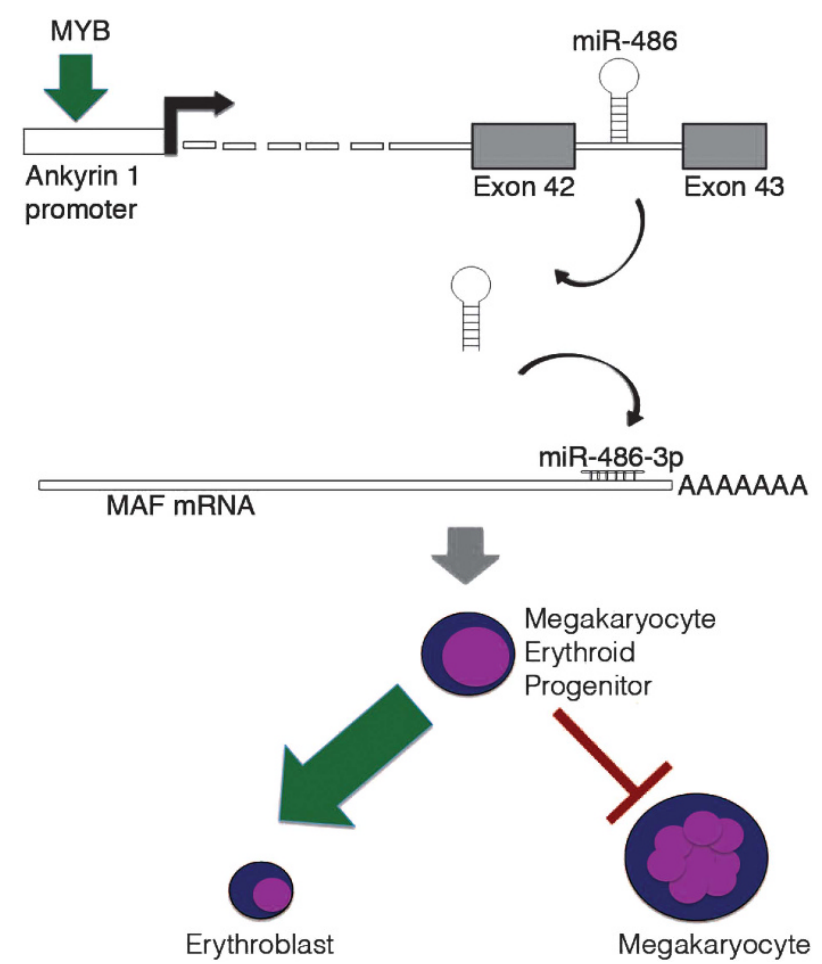

Figure 7 Schematic illustration of the mechanism through which the MYB/ miR-486-3p/MAF axis regulates erythroid versus megakaryocyte lineage fate decision. MYB-mediated transactivation of ANK1 expression determines the upregulation of miR-486-3p, which in turn targets MAF mRNA for degradation. Therefore, through the downregulation of MAF, MYB restrains the megakaryocyte commitment and favors the erythroid one in megakaryocyte erythroid progenitors 
cells were seeded $\left(5 \times 10^{5} / \mathrm{ml}\right)$ in IMDM added with $20 \%$ BIT serum substitute (bovine serum albumin, insulin and transferrin; StemCell Technologies) in order to set up megakaryocyte (THPO $50 \mathrm{ng} / \mathrm{ml}$, SCF $10 \mathrm{ng} / \mathrm{ml}$, adapted from Tenedini et al..$^{32}$ ), granulocyte (GCSF $25 \mathrm{ng} / \mathrm{ml}$, SCF $10 \mathrm{ng} / \mathrm{ml}$, adapted from Kandilci et $\mathrm{al} . .^{33}$ ) and monocyte (MCSF $100 \mathrm{ng} / \mathrm{ml}$, SCF $20 \mathrm{ng} / \mathrm{ml}$, IL6 $20 \mathrm{ng} / \mathrm{ml}$ and Flt3L $50 \mathrm{ng} / \mathrm{ml}^{33}$ ) unilineage cultures (all cytokines from Miltenyi Biotec). The medium was replaced every 3 days. CD34+ cells differentiation was monitored by by morphological analysis of MGG-stained cytospins and by flow-cytometric analysis of differentiation marker expression. miR-486-3p and MAF expression levels were detected by qRT-PCR at different time points (i.e. days $0,2,4,6$ and 8) after the seeding of cells in megakaryocyte, granulocyte or mono-macrophage unilineage cultures.

Quantitative reverse transcription polymerase chain reaction. Total RNA (100 ng) was reverse-transcribed to cDNA using a High Capacity cDNA Archive Kit (Life Technologies). TaqMan PCR was carried out by using the TaqMan Fast Advanced PCR master mix and TaqMan gene expression assays (all reagents from Life Technologies), by means of a $7900 \mathrm{HT}$ Fast Real-Time PCR System (Applied Biosystems). Assays were performed in triplicate. Gene expression profiling was achieved using the comparative cycle threshold (CT) method of relative quantitation using hypoxanthine phosphoribosyltransferase 1 (HPRT1) as the housekeeping gene. To normalize data, $\Delta \Delta C T$ was calculated for each sample using the mean of its $\Delta \mathrm{CT}$ values subtracted from the mean $\Delta \mathrm{CT}$ value measured in the MOCK sample, set as a calibrator; relative quantitation (RQ) value was expressed as $2^{-\Delta \Delta C T}$.

Individual miRNA detection by RT-qPCR was performed using the TaqMan MicroRNA assays (Life technologies). Individual reverse transcription reactions ( $5 \mathrm{ng}$ of total RNA each target) were performed using the Taqman microRNA Reverse Transcription Kit and the miRNA-specific looped-primers. TaqMan PCR was performed in triplicate by using the $7900 \mathrm{HT}$ Fast Real-Time PCR System (Applied Biosystems). miRNA expression $R Q$ data were calculated as reported above, by using SNORD49A as the housekeeping control.

Chromatin ImmunoPrecipitation. ChIP assay was performed as reported by Lang et al. ${ }^{34}$ with some modifications. After crosslinking in $1 \%$ formaldehyde, highly proliferating CB-derived CD34+ cells $\left(10 \times 10^{6}\right.$ cells/sample) were resuspended in $2 \mathrm{ml}$ lysis buffer ( $5 \mathrm{mM}$ PIPES, $85 \mathrm{mM} \mathrm{KCl}, 0.5 \%$ NP-40, protease inhibitors (Roche), $1 \mathrm{mM}$ PMSF) and disrupted with a Dounce homogenizer, then incubated on ice for $10 \mathrm{~min}$. Nuclei were pelleted and resuspended in $1 \mathrm{ml}$ sonication buffer $(0.1 \%$ SDS, $10 \mathrm{mM}$ EDTA pH $8,50 \mathrm{mM}$ Tris $\mathrm{pH}$ 8, protease inhibitors (Roche), $1 \mathrm{mM}$ PMSF) and incubated on ice for $10 \mathrm{~min}$. Chromatin with an average size of $500-1000 \mathrm{bp}$ was produced by sonication. Debris were cleared by centrifugation. Chromatin (100 $\mu$ l for each sample) was diluted twofold into RIPA buffer (10 mM TrisHCl pH8, 1 mM EDTA pH8, 0.5 mM EGTA, 1\%Triton X-100, 0,1\% Na-deoxycholate, $0,1 \%$ SDS, $140 \mathrm{mM} \mathrm{NaCl}, 1 \mathrm{mM}$ PMSF and protease inhibitors (Roche) and pre-cleared with Protein G Agarose slurry (KPL, Gaithersburg, MD, USA), then immunoprecipitated with (MYB sample) or without (no-antibody sample) mouse monoclonal anti-MYB antibody (Anti-MYB, clone 1-1, catalog \#27282, Upstate, Lake Placid, NY) overnight at $4{ }^{\circ} \mathrm{C}$ with rotation. A negative control (named IgG CTR) sample incubated with an isotype-matched non-specific antibody (Upstate, Lake Placid, NY, USA) was included. Protein G Agarose slurry (KPL) was pre-cleared in RIPA buffer with $1 \mu \mathrm{g} / \mu \mathrm{l}$ salmon sperm DNA and $1 \mu \mathrm{g} / \mu \mathrm{l}$ BSA overnight at $4{ }^{\circ} \mathrm{C}$ with rotation and then washed twice in ChIP buffer. Immune complexes were collected by incubation with pre-cleared protein $\mathrm{G}$ agarose for $2 \mathrm{~h}$ with rotation at $4^{\circ} \mathrm{C}$. The supernatant fraction of the no-antibody sample was collected as total input chromatin. The protein $\mathrm{G}$ agarose/antibody/chromatin complex was washed five-fold with RIPA buffer, then once with LiCl buffer buffer (250 mM LiCl, 0.5\%NP40, 0.5\% Na deoxycholate, $1 \mathrm{mM}$ EDTA, $10 \mathrm{mM}$ TrisHCl pH8) and TE buffer ( $1 \mathrm{mM}$ EDTA, $10 \mathrm{mM}$ TrisHCl pH8) respectively. Beads were resuspended in $100 \mu \mathrm{l}$ of TE buffer. Samples were incubated with RNase A overnight at $65^{\circ} \mathrm{C}$, then with $0.5 \%$ SDS and $0.5 \mathrm{mg} / \mathrm{ml}$ proteinase $\mathrm{K}$ at $50^{\circ} \mathrm{C}$ for $3 \mathrm{~h}$. Immunoprecipitated DNA samples were isolated by phenol-chloroform extraction, precipitated in ice-cold ethanol at $-20^{\circ} \mathrm{C}$ and resuspended in $30 \mu \mathrm{l}$ of distilled water. Primers for PCR were as follows: ANK1 $(-1292 /-1287)$ forward: gaagctgatctcggtggcat; ANK1 (-1292/-1287) reverse: cgtgatgcttggcctcctg; ANK1 (-1008/999) forward: ggaaaccatggagggagaca; ANK1 (-1008/999) reverse: gtccaacaacgggatgagatg; CTSG forward: TTG ATG GGT TGA GGG TTC TGG; CTSG reverse: CAG TCA GTT GCT GCT GTG CTT C. CTSG promoter-specific PCR was included as a positive control for MYB antibody-mediated ChIP, since CTSG has already been demonstrated to be a MYB target gene. ${ }^{35}$ ChIP reactions were done at least twice for each preparation using three different preparations of CD34+ cells. Data shown are for 32 cycles.

Luciferase reporter assay. The human ANK1 promoter reporter construct was made by cloning the proximal $-1498 /+47$ fragment of ANK1 promoter into the promoterless vector $\mathrm{PXP} 1$ containing Firefly luciferase reporter gene.

The human ANK1 promoter - 1498/+47 clone was made by amplifying the PCR product by using forward CGCGGATCCGCGTTAAGGACTGTGCTGTGGTAGA (BamHI) and reverse GGAAGATCTTCCGGGCTTGAGGAGGAGCA (BgllI) primers, cutting with the restriction enzymes BamHI and Bglll and inserting it into pXP1 vector using these sites.

The cloned fragment for ANK1 promoter region was amplified from genomic DNA of human CB CD34+ cells and originally inserted in pCR2.1 vector (Invitrogen). The sequences of all promoter constructs were verified by sequencing.

Approximately $1-1.5 \times 10^{5}$ HEK293T cells were transiently transfected with Lipofectamine 2000 (Invitrogen) using 100 to $400 \mathrm{ng}$ of pCMV6XL5-MYB vector (Origene, Rockville, MD), $200 \mathrm{ng}$ of ANK1-Firefly luciferase, $2 \mathrm{ng}$ of pGL4.75 hRLuc/ CMV (Promega, Madison, WI, USA), and carrier plasmid to level the total amount of transfected DNA for all the samples. Firefly and Renilla luciferase activity was measured $48 \mathrm{~h}$ after the transfection using the Dual-Luciferase Reporter Assay System (Promega) and luminescence was recorded on a GloMax-Multi+ Detection System with Intinct Software (Promega), according to the manufacturer's protocol. Five independent experiments were performed in duplicate. Firefly luciferase activity was normalized to that of Renilla luciferase.

CD34+ cells culture conditions. After immunomagnetic separation, CD34+ cells were seeded in 24-well plates at $5 \times 10^{5} / \mathrm{ml}$ in IMDM (Euroclone) containing $20 \%$ human serum (Bio-Whittaker), stem cell factor (SCF; $50 \mathrm{ng} / \mathrm{ml}$ ), Fms-like tyrosine kinase 3 ligand (Flt3L; $50 \mathrm{ng} / \mathrm{ml}$ ), thrombopoietin (THPO; $20 \mathrm{ng} / \mathrm{ml}$ ), interleukin-6 (IL-6; $10 \mathrm{ng} / \mathrm{ml}$ ) and interleukin-3 (IL-3; $10 \mathrm{ng} / \mathrm{ml}$; all from Miltenyi).

After each transfection, cells were transferred into pre-warmed fresh medium in 24well plates and maintained in the same culture conditions as described above.

For liquid culture differentiation assays, $24 \mathrm{~h}$ after the last nucleofection (hereafter reported as post nucleofection) CD34+ cells were plated $\left(5 \times 10^{5} / \mathrm{ml}\right)$ in IMDM added with $20 \%$ BIT serum substitute (bovine serum albumin, insulin, and transferrin; StemCell Technologies), in order to set up erythroid (erythropoietin (EPO) $0.4 \mathrm{U} / \mathrm{ml}$, SCF $10 \mathrm{ng} / \mathrm{ml}$, adapted from Bianchi et al., ${ }^{3}$ megakaryocyte (THPO $50 \mathrm{ng} / \mathrm{ml}$, SCF $10 \mathrm{ng} / \mathrm{ml}$, adapted from Tenedini et al..$^{32}$ ), granulocyte (GCSF $25 \mathrm{ng} / \mathrm{ml}$, SCF $10 \mathrm{ng} / \mathrm{ml}$, adapted from Kandilci et al. ${ }^{33}$ ) and monocyte (MCSF $100 \mathrm{ng} / \mathrm{ml}$, SCF $20 \mathrm{ng} / \mathrm{ml}$, IL6 $20 \mathrm{ng} / \mathrm{ml}$ and Flt3L $50 \mathrm{ng} / \mathrm{ml} ;{ }^{33}$ all cytokines from Miltenyi Biotec) unilineage cultures. The medium was replaced every 3 days.

Morphological and immunophenotypic analysis. Differentiation of CD34+ cells was assessed by morphological analysis of MGG-stained cytospins at 8,11 and 13 days post nucleofection and by flow-cytometric analysis of differentiation markers expression (CD34, CD71, CD36, Glycophorin A (GPA), CD15, myeloperoxydase (MPO), CD14, CD163, CD41, CD42b) at 4, 8, 10 and 12 days post nucleofection.

The images were captured by using an Ax10scopeA1 microscope equipped with an AxioCam ERc 5S Digital Camera and Axion software 4.8 (all Carl Zeiss Microlmaging Inc.; Thornwood, NY, USA). The images were then processed with Adobe Photoshop 7.0 software.

The following monoclonal antibodies (MoAbs) were used for flow-cytometric analysis: fluorescein isothiocyanate (FITC)-conjugated mouse anti-CD41 MoAb, $\mathrm{PE}$-conjugated mouse anti-human CD42b MoAb, PE-conjugated mouse anti-human GPA MoAb (all from Dako; Milano, Italia; http://www.dako.com), VioBlue-conjugated mouse anti-CD34 MoAb, FITC-conjugated mouse anti-human CD15 MoAb, FITCconjugated mouse anti-human CD36 MoAb, allophycocyanin (APC)-conjugated mouse anti-human CD71 MoAb, PE-conjugated mouse anti-human CD14 MoAb, APC-conjugated mouse anti-human CD163 MoAb (all from Miltenyi Biotec) and FITC-conjugated mouse anti-human MPO MoAb (from BD Biosciences; San Jose, CA USA). After staining, cells were analyzed by using a BD FACSCanto II (BD Biosciences). At least 10000 events were counted for each sample to ensure statistical relevance.

Collagen-based megakaryocyte clonogenic assay. CFU-MK were assayed in collagen-based medium, by using a commercial Mk assay detection kit (MegaCult-C, StemCell Technologies) as previously described. ${ }^{3}$ CFU-MKs were scored according to the manufacturer's protocol as small (3-21 cells, deriving from 
more mature megakaryocyte progenitors), medium (21-49 cells) and large ( $>50$ cells, arising from more primitive MK progenitors) colonies based on their size, which reflects the maturation stage of the progenitor giving rise to each colony.

$3^{\prime}$ Untranslated region ( $3^{\prime}$ UTR) luciferase reporter assays. Fulllength $3^{\prime}$ UTR from MAF variant 1 (RefSeq NM_005360.3) and MAF variant 2 (NM_001031804.1; given its size, the latter $3^{\prime}$ UTR was cloned in two fragments, 1-2569 bps and 2530-4932 bps, respectively) transcripts and empty pEZX-MT01 luciferase reporter constructs were purchased from Labomics (Genecopoeia; MD, USA). Every plasmid contains the firefly luciferase gene upstream of a given $3^{\prime}$ untranslated region $\left(3^{\prime} \cup T R\right)$, and the Renilla luciferase gene acting as a normalizer gene.

K562 cells were electroporated by means of the Amaxa 4D-Nucleofector System (Lonza Group Ltd), according to the manufacturer's instructions. Briefly, K562 cells were co-nucleofected with either a miR-486-3p mimic or NegCTR mimic at a concentration of $3.6 \mu \mathrm{M}$ and with either $3^{\prime} U T R$-less luciferase or a full-length $3^{\prime} U T R$ construct at a concentration of $0.4 \mathrm{pmol} / \mathrm{sample}$. For each electroporation, $10^{6}$ cells were resuspended in $100 \mu \mathrm{l}$ of SF Cell line Solution (Lonza Group Ltd) and pulsed with the program FF120. Firefly and Renilla luciferase activities were measured at 24 , 48 and $72 \mathrm{~h}$ after nucleofection using the Dual-Luciferase Reporter Assay System (Promega), and luminescence was recorded on a GloMax-Multi+ Detection System with Intinct Software (Promega), according to the manufacturer's protocol.

Firefly luciferase activity was first normalized to that of Renilla luciferase as an internal control for nucleofection efficiency. Next, for each $3^{\prime} U T R$ luciferase reporter construct the luminescence signal was normalized to the effect of the NegCTR mimic-transfected sample.

Western blot. MAF protein levels in both miR-486-3p/NegCTR mimictransfected and in LMAFvar2IDN/LXIDN-transduced CD34+ cells were detected by western blot analysis. Briefly: cells were harvested, washed twice with ice-cold PBS and lysed $\left(5 \times 10^{5} \mathrm{cells} / 20 \mu \mathrm{l}\right.$ of lysis buffer) in $50 \mathrm{mM}$ Tris (tris(hydroxymethyl) aminomethane)- $\mathrm{Cl}$ (pH 7.4), $150 \mathrm{mM} \mathrm{NaCl}, 1 \%$ Nonidet P-40, $10 \mathrm{mM} \mathrm{KCl}, 1 \mathrm{mM}$ EDTA, $20 \mathrm{mM} \mathrm{NaF}, 0.25 \%$ Na doexycholate, $5 \mathrm{mM}$ dithiothreitol (DTT) and protease inhibitors (Complete, catalog \#1697498, Roche). Total cellular lysates (15 $\mu \mathrm{g}$ for each sample) were loaded onto $12.5 \%$ SDS-polyacrylamide gel and blotted on nitrocellulose membrane. To control loading and transfer, after transfer the membranes were stained by Red Ponceau. Membranes were then preblocked in blocking solution, $5 \%$ milk in $0.1 \%$ TBST for $1 \mathrm{~h}$ at room temperature (RT), incubated with primary antibodies $(1: 2000$ dilution of rabbit monoclonal anti-MAF antibody (1:2000 dilution, catalog \#181188, Abcam, Cambridge, UK; www.abcam.com), at $4^{\circ} \mathrm{C}$ overnight of rabbit polyclonal anti-actin antibody (1: 5000 dilution, Thermo Fisher Scientific Inc., Waltham, MA, USA, catalog \#PA116889) for $1 \mathrm{~h}$ at RT. After three washes with TBST, blots were incubated with HRPconjugated goat anti-rabbit secondary antibody $(1: 1000$ dilution, Thermo Fisher Scientific Inc.) for $1 \mathrm{~h}$ at RT and revealed by BM Chemiluminescence Blotting Substrate (POD) (Roche).

CD34+ cells transduction and purification. In order to achieve the overexpression of both miR-486-3p and MAF in human progenitor cells (HPCs), CD34+ cells were nucleofected with miR-486-3p/NegCTR mimic as already described in 'nucleofection of CD34+ cells'. Next, $12 \mathrm{~h}$ after the second nucleofection, CD34+ cells were transduced in 24-wells plates at a density of $5 \times 105 / \mathrm{ml}$ in serum-free medium SYN-H (ABCell-Bio, Paris, France) supplemented with SCF $(50 \mathrm{ng} / \mathrm{ml})$, FLT3L $(50 \mathrm{ng} / \mathrm{ml})$, THPO $(20 \mathrm{ng} / \mathrm{ml})$, IL-3 $(10 \mathrm{ng} / \mathrm{ml})$ and IL-6 $(10 \mathrm{ng} / \mathrm{ml}$; all cytokines from Miltenyi Biotec). CD34+ cells were transduced by means of three cycles of infection (12 $\mathrm{h}$ each) with viral supernatant in the presence of polybrene $(8 \mu \mathrm{g} / \mathrm{ml})$ in retronectin-coated plates $(10 \mu \mathrm{g} / \mathrm{cm} 2)$. After transduction, cells were maintained in the same culture conditions reported above for $36 \mathrm{~h}$.

Transduction efficiency was monitored by flow-cytometric detection of the NGFRpositive (NGFR+) fraction after labeling with a PE-conjugated anti-NGFR antibody (Miltenyi Biotec). Transduced CD34+ cells were subsequently purified by an immunomagnetic procedure (EasySep 'Do-It-Yourself' Selection Kit; StemCell Technologies) by means of a anti-human p75-NGFR mouse monoclonal antibody (BD Biosciences) and seeded in the liquid culture conditions as reported above.

NGFR+ cells were analyzed for both MAF and miR-486-3p expression by qRTPCR. CFU-MK were assayed by plating 10000 NGFR+ cells for sample in collagenbased medium, as already described above. For liquid culture differentiation assays, NGFR+ cells were plated ( $5 \times 105 / \mathrm{ml}$ ) in: (a) serum-free medium SYN-H (ABCell-Bio, Paris, France) supplemented with SCF (5 ng/ml), THPO (50 ng/ml), IL11 (40 ng/ml),
IL3 $(2 \mathrm{ng} / \mathrm{ml})$ and IL6 $(1 \mathrm{ng} / \mathrm{ml})$ in order to support the differentiation towards the megakaryocyte lineage; (b) IMDM added with $20 \%$ BIT serum substitute (bovine serum albumin, insulin, and transferrin; StemCell Technologies) supplemented with erythropoietin (EPO; $0.4 \mathrm{U} / \mathrm{ml}$; Neorecormon, Roche) and SCF (10 ng/ml; Miltenyi) in order to support the erythroid differentiation. The medium was replaced every 2 days. Differentiation was assessed by morphological analysis of MGG-stained cytospins and by flow-cytometric analysis of CD41, CD42b, CD71, CD36 and GPA differentiation markers.

Statistical analysis. The statistics used for data analysis in silencing/overexpression experiments and luciferase reporter assays were based on two-tailed Student's t-tests for averages comparison in paired samples. Data were analyzed by using Microsoft Excel (Microsoft Office, 2008 release, Microsoft, Redmont, WA, USA) and are reported as mean \pm S.E.M.. $P<0.05$ was considered significant.

\section{Conflict of Interest}

The authors declare no conflict of interest.

Acknowledgements. This work was supported by Associazione Italiana per la Ricerca sul Cancro (AIRC), project number \#10005 'Special Program Molecular Clinical Oncology $5 \times 1000$ ' to AGIMM (AIRC-Gruppo Italiano Malattie Mieloproliferative, http://www.progettoagimm.it); AIRC project number \#12055; Italian Ministry of University \& Research (FIRB Project 2011, project number \#RBAP11CZLK, and PRIN 2010-11, project number 2010NYKNS7); Project Tecnopolo (Regione Emilia Romagna http://www.aster.it/tiki-index.php?page=Tecnopoli).

\section{Author contributions}

EB performed gene/miRNA silencing and overexpression experiments, and wrote the paper; JB performed GEP/miEP integrative analysis and ANK1 promoter luciferase assays; SR and VP performed array hybridization and miR-486-3p and MAF kinetics during differentiation; SR performed $3^{\prime} U T R$ reporter assays and western blot analysis; GS performed miRNA profiling and miEP data analysis; RN performed analysis of GEP upon MYB silencing; RZ performed the computational analysis of putative MYB targets in ANK1 promoter; SS performed Chromatin Immunoprecipitation; ZP performed miR-486-3p and ANK1 kinetics during erythroid differentiation; SF and RM designed the research and wrote the paper.

1. Mucenski ML, McLain K, Kier AB, Swerdlow SH, Schreiner CM, Miller TA et al. A functional c-myb gene is required for normal murine fetal hepatic hematopoiesis. Cell 1991; 65: $677-689$.

2. Emambokus N, Vegiopoulos A, Harman B, Jenkinson E, Anderson G, Frampton J. Progression through key stages of haemopoiesis is dependent on distinct threshold levels of c-Myb. Embo J 2003; 22: 4478-4488.

3. Bianchi E, Zini R, Salati S, Tenedini E, Norfo R, Tagliafico E et al. c-myb supports erythropoiesis through the transactivation of KLF1 and LMO2 expression. Blood 2010; 116: e99-110.

4. Carpinelli MR, Hilton DJ, Metcalf D, Antonchuk JL, Hyland CD, Mifsud SL et al. Suppressor screen in Mpl-- mice: c-Myb mutation causes supraphysiological production of platelets in the absence of thrombopoietin signaling. Proc Natl Acad Sci USA 2004; 101: 6553-6558.

5. Kauppi M, Murphy JM, de Graaf CA, Hyland CD, Greig KT, Metcalf D et al. Point mutation in the gene encoding p300 suppresses thrombocytopenia in Mpl-/- mice. Blood 2008; 112: 3148-3153.

6. Metcalf D, Carpinelli MR, Hyland C, Mifsud S, Dirago L, Nicola NA et al. Anomalous megakaryocytopoiesis in mice with mutations in the c-Myb gene. Blood 2005; 105 : 3480-3487.

7. Bouilloux F, Juban G, Cohet N, Buet D, Guyot B, Vainchenker W et al. EKLF restricts megakaryocytic differentiation at the benefit of erythrocytic differentiation. Blood 2008; 112 : 576-584.

8. Frontelo P, Manwani D, Galdass M, Karsunky H, Lohmann F, Gallagher PG et al. Novel role for EKLF in megakaryocyte lineage commitment. Blood 2007; 110: 3871-3880.

9. Warren AJ, Colledge WH, Carlton MB, Evans MJ, Smith AJ, Rabbitts TH. The oncogenic cysteine-rich LIM domain protein rbtn2 is essential for erythroid development. Cell 1994; 78: 45-57.

10. Cao S, Liu J, Song L, Ma X. The protooncogene c-Maf is an essential transcription factor for IL-10 gene expression in macrophages. J Immunol 2005; 174: 3484-3492.

11. Hegde SP, Zhao J, Ashmun RA, Shapiro LH. c-Maf induces monocytic differentiation and apoptosis in bipotent myeloid progenitors. Blood 1999; 94: 1578-1589.

12. Sevinsky JR, Whalen AM, Ahn NG. Extracellular signal-regulated kinase induces the megakaryocyte GPIlb/CD41 gene through MafB/Kreisler. Molecular and cellular biology 2004; 24: 4534-4545. 
13. Azcoitia V, Aracil M, Martinez AC, Torres M. The homeodomain protein Meis1 is essential for definitive hematopoiesis and vascular patterning in the mouse embryo. Dev Biol 2005; 280: 307-320.

14. Chen CZ, Li L, Lodish HF, Bartel DP. MicroRNAs modulate hematopoietic lineage differentiation. Science 2004; 303: 83-86.

15. Zhang L, Sankaran VG, Lodish HF. MicroRNAs in erythroid and megakaryocytic differentiation and megakaryocyte-erythroid progenitor lineage commitment. Leukemia 2012; 26: 2310-2316.

16. Lee RC, Feinbaum RL, Ambros V. The $C$. elegans heterochronic gene lin-4 encodes small RNAs with antisense complementarity to lin-14. Cell 1993; 75: 843-854.

17. Norfo R, Zini R, Pennucci V, Bianchi E, Salati S, Guglielmelli $P$ et al. miRNA-mRNA integrative analysis in primary myelofibrosis CD34+ cells unveils the role of miR-155/JARID2 axis in abnormal megakaryopoiesis. Blood 2014; 124: e21-e32.

18. Hitachi K, Nakatani M, Tsuchida K. Myostatin signaling regulates Akt activity via the regulation of miR-486 expression. Int J Biochem Cell Biol 2014; 47: 93-103.

19. Alexander MS, Casar JC, Motohashi N, Myers JA, Eisenberg I, Gonzalez RT et al. Regulation of DMD pathology by an ankyrin-encoded miRNA. Skelet Muscle 2011; 1 27.

20. Shaham L, Vendramini E, Ge Y, Goren Y, Birger Y, Tijssen MR et al. MicroRNA-486-5p is an erythroid oncomiR of the myeloid leukemias of Down syndrome. Blood 2015; 125 : $1292-1301$.

21. Heinemeyer T, Wingender E, Reuter I, Hermjakob H, Kel AE, Kel OV et al. Databases on transcriptional regulation: TRANSFAC, TRRD and COMPEL. Nucleic Acids Res 1998; 26 : 362-367.

22. Hedge SP, Kumar A, Kurschner C, Shapiro LH. c-Maf interacts with c-Myb to regulate transcription of an early myeloid gene during differentiation. Mol Cell Biol 1998; 18 . 2729-2737.

23. Peng S, Lalani S, Leavenworth JW, Ho IC, Pauza ME. c-Maf interacts with c-Myb to downregulate Bcl-2 expression and increase apoptosis in peripheral CD4 cells. Eur J Immunol 2007; 37: 2868-2880.

24. Lu J, Guo S, Ebert BL, Zhang H, Peng X, Bosco J et al. MicroRNA-mediated control of cell fate in megakaryocyte-erythrocyte progenitors. Dev Cell 2008; 14: 843-853.

25. Barroga CF, Pham H, Kaushansky K. Thrombopoietin regulates c-Myb expression by modulating micro RNA 150 expression. Exp Hematol 2008; 36: 1585-1592.

26. Navarro F, Gutman D, Meire E, Caceres M, Rigoutsos I, Bentwich Z et al. miR-34a contributes to megakaryocytic differentiation of K562 cells independently of p53. Blood 2009; 114: 2181-2192.
27. Sankaran VG, Menne TF, Scepanovic D, Vergilio JA, Ji P, Kim J et al. MicroRNA-15a and $-16-1$ act via MYB to elevate fetal hemoglobin expression in human trisomy 13. Proc Natl Acad Sci USA 2011; 108: 1519-1524.

28. Kamat V, Paluru P, Myint M, French DL, Gadue P, Diamond SL. MicroRNA screen of human embryonic stem cell differentiation reveals miR-105 as an enhancer of megakaryopoiesis from adult CD34+ cells. Stem Cells 2014; 32: 1337-1346.

29. Wang LS, Li L, Li L, Chu S, Shiang KD, Li M et al. MicroRNA-486 regulates normal erythropoiesis and enhances growth and modulates drug response in $\mathrm{CML}$ progenitors. Blood 2015; 125: 1302-1313.

30. Edgar R, Domrachev M, Lash AE. Gene expression omnibus: NCBI gene expression and hybridization array data repository. Nucleic Acids Res 2002; 30: 207-210.

31. Andersen $\mathrm{CL}$, Jensen JL, Orntoft TF. Normalization of real-time quantitative reverse transcription-PCR data: a model-based variance estimation approach to identify genes suited for normalization, applied to bladder and colon cancer data sets. Cancer Res 2004; 64: $5245-5250$

32. Tenedini E RE, Ferrari F, Orlandi C, Bianchi E, Bicciato $S$ et al. Integrated analysis of microRNA and mRNA expression profiles in physiological myelopoiesis: role of hsa-mir-299-5p in CD34+ progenitor cells commitment. Cell Death Dis 2010; 1: e28.

33. Kandilci A, Grosveld GC. Reintroduction of CEBPA in MN1-overexpressing hematopoietic cells prevents their hyperproliferation and restores myeloid differentiation. Blood 2009; 114: 1596-1606.

34. Lang G, White JR, Argent-Katwala MJ, Allinson CG, Weston K. Myb proteins regulate the expression of diverse target genes. Oncogene 2005; 24: 1375-1384.

35. Lennartsson A, Garwicz D, Lindmark A, Gullberg U. The proximal promoter of the human cathepsin $\mathrm{G}$ gene conferring myeloid-specific expression includes C/EBP, c-myb and PU.1 binding sites. Gene 2005; 356: 193-202.

(c) (i) $(9)($ This work is licensed under a Creative Commons Attribution-NonCommercial-NoDerivs 4.0 International License. The images or other third party material in this article are included in the article's Creative Commons license, unless indicated otherwise in the credit line; if the material is not included under the Creative Commons license, users will need to obtain permission from the license holder to reproduce the material. To view a copy of this license, visit http://creativecommons.org/licenses/by-nc-nd/4.0/

Supplementary Information accompanies this paper on Cell Death and Differentiation website (http://www.nature.com/cdd) 